\title{
Forward doubly-virtual Compton scattering off the nucleon in chiral perturbation theory. II. Spin polarizabilities and moments of polarized structure functions
}

\author{
Jose Manuel Alarcón๑* \\ Departamento de Física Teórica \& IPARCOS, Universidad Complutense de Madrid, 28040 Madrid, Spain \\ Franziska Hagelstein $\odot$ \\ Albert Einstein Center for Fundamental Physics, Institute for Theoretical Physics, \\ University of Bern, Sidlerstrasse 5, CH-3012 Bern, Switzerland \\ Vadim Lensky॰ and Vladimir Pascalutsa® \\ Institut für Kernphysik \& Cluster of Excellence PRISMA, Johannes Gutenberg-Universität Mainz, \\ D-55128 Mainz, Germany
}

(Received 20 June 2020; accepted 22 September 2020; published 17 December 2020)

\begin{abstract}
We examine the polarized doubly-virtual Compton scattering (VVCS) off the nucleon using chiral perturbation theory $(\chi \mathrm{PT})$. The polarized VVCS contains a wealth of information on the spin structure of the nucleon which is relevant to the calculation of the two-photon-exchange effects in atomic spectroscopy and electron scattering. We report on a complete next-to-leading-order calculation of the polarized VVCS amplitudes $S_{1}\left(\nu, Q^{2}\right)$ and $S_{2}\left(\nu, Q^{2}\right)$, and the corresponding polarized spin structure functions $g_{1}\left(x, Q^{2}\right)$ and $g_{2}\left(x, Q^{2}\right)$. Our results for the moments of polarized structure functions, partially related to different spin polarizabilities, are compared to other theoretical predictions and "data-driven" evaluations, as well as to the recent Jefferson Lab measurements. By expanding the results in powers of the inverse nucleon mass, we reproduce the known "heavy-baryon" expressions. This serves as a check of our calculation, as well as demonstrates the differences between the manifestly Lorentz-invariant baryon $\chi \mathrm{PT}$ and heavy-baryon frameworks.
\end{abstract}

DOI: $10.1103 /$ PhysRevD.102.114026

\section{INTRODUCTION}

In the studies of nucleon structure, the forward doublyvirtual Compton scattering (VVCS) amplitude, Fig. 1, is playing a central role (see, e.g., Refs. [1-4] for reviews). Traditionally, its general properties, such as unitarity, analyticity, and crossing, are used to establish various useful sum rules for the nucleon magnetic moment (Gerasimov-Drell-Hearn [5,6] and Schwinger sum rules [7-9]) and polarizabilities (e.g., Baldin [10] and Gell-Mann-Goldberger-Thirring sum rules [11]). More recently, the interest in nucleon VVCS has been renewed in connection with precision atomic spectroscopy, where this amplitude enters in the form of two-photon exchange (TPE) corrections. As the TPE corrections in the atomic

\footnotetext{
*jmalarcon@ucm.es
}

Published by the American Physical Society under the terms of the Creative Commons Attribution 4.0 International license. Further distribution of this work must maintain attribution to the author(s) and the published article's title, journal citation, and DOI. Funded by SCOAP. domain are dominated by low-energy VVCS, it makes sense to calculate them systematically using chiral perturbation theory $(\chi \mathrm{PT})$, which is a low-energy effective-field theory of the Standard Model.

In this paper, we present a state-of-the-art $\chi \mathrm{PT}$ calculation of the polarized nucleon VVCS, relevant to TPE corrections to the hyperfine structure of hydrogen and muonic hydrogen. This will extend the leading-order $\chi \mathrm{PT}$ evaluation of the TPE effects in hyperfine splittings [12-17]. Here, however, we do not discuss the TPE evaluation, but rather focus on testing the $\chi \mathrm{PT}$ framework against the available empirical information on the lowenergy spin structure of the nucleon.

It is especially interesting to confront the $\chi \mathrm{PT}$ predictions with the recent measurements coming from the ongoing "Spin Physics Program" at Jefferson Laboratory [18-27], with the exception of a recent measurement of the deuteron spin polarizability by the CLAS Collaboration [28], which does not treat correctly complications due to deuteron spin [29].

Our present calculation is extending Ref. [30] to the case of polarized VVCS. We therefore use a manifestly 


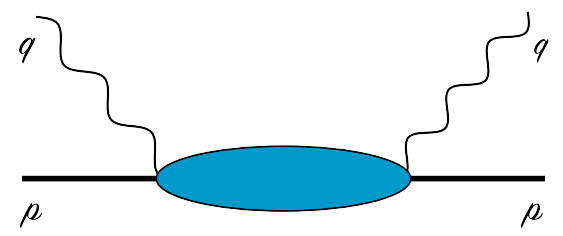

FIG. 1. The forward Compton scattering, or VVCS, in the case of virtual photons, $q^{2}=-Q^{2}$.

covariant extension of $\mathrm{SU}(2) \chi \mathrm{PT}$ to the baryon sector called baryon $\chi \mathrm{PT}(\mathrm{B} \chi \mathrm{PT})$. First attempts to calculate VVCS in the straightforward $\mathrm{B} \chi \mathrm{PT}$ framework (rather than the heavy-baryon expansion or the "infrared regularization") were done by Bernard et al. [31] and our group [32]. The two works obtained somewhat different results, most notably for the proton spin polarizability $\delta_{L T}$. Here we improve on [32] in three important aspects appreciable at finite $Q^{2}$ : (1) inclusion of the Coulomb-quadrupole $(C 2)$ $N \rightarrow \Delta$ transition [33,34]; (2) correct inclusion of the elastic form-factor contributions to the integrals $\Gamma_{1}\left(Q^{2}\right)$, $I_{1}\left(Q^{2}\right)$, and $I_{A}\left(Q^{2}\right)$ (see Secs. III C and III D for details), and (3) cancellations between different orders in the chiral prediction and their effect on the convergence of the effective-field-theory calculation, and thus, the error estimate. These improvements, however, do not bring us closer to the results of [31], and the source of discrepancies most likely lies in the different counting and renormalization of the $\pi \Delta$-loop contributions. Bernard et al. [31] use the socalled small-scale expansion [35] for the $\Delta(1232)$ contributions, whereas we are using the $\delta$-counting scheme [36] (see also Ref. [37] [Sec. 4] for review).

This paper is organized as follows. In Sec. II A, we introduce the polarized VVCS amplitudes and their relations to spin structure functions. In Sec. II B, we introduce the spin polarizabilities appearing in the low-energy expansion (LEX) of the polarized VVCS amplitudes. In Sec. II C, we briefly describe our $\chi \mathrm{PT}$ calculation, focusing mainly on the uncertainty estimate. In Sec. III, we show our predictions for the proton and neutron polarizabilities, as well as some interesting moments of their structure functions. In Sec. III G, we summarize the results obtained herein, comment on the improvements done with respect to previous calculations, and give an outlook to future applications. In Appendix B, we discuss the structure functions; in particular, we define the longitudinaltransverse response function, discuss the $\Delta$-pole contribution, and give analytical results for the tree-level $\pi N$ - and $\Delta$-production channels of the photoabsorption cross sections. In Appendix $\mathrm{C}$, we give analytical expressions for the $\pi N$-loop and $\Delta$-exchange contributions to the central values and slopes of the polarizabilities and moments of structure functions at $Q^{2}=0$. The complete expressions, also for the $\pi \Delta$-loop contributions, can be found in the Supplemental Material [38].

\section{CALCULATION OF UNPOLARIZED VVCS AT NLO}

\section{A. VVCS amplitudes and relations to structure functions}

The polarized part of forward VVCS can be described in terms of two independent Lorentz-covariant and gaugeinvariant tensor structures and two scalar amplitudes [3]:

$$
\begin{aligned}
T^{\mu \nu}(p, q) & \\
= & -\frac{1}{M_{N}} \gamma^{\mu \nu \alpha} q_{\alpha} S_{1}\left(\nu, Q^{2}\right) \\
& \quad-\frac{1}{M_{N}^{2}}\left(\gamma^{\mu \nu} q^{2}+q^{\mu} \gamma^{\nu \alpha} q_{\alpha}-q^{\nu} \gamma^{\mu \alpha} q_{\alpha}\right) S_{2}\left(\nu, Q^{2}\right) .
\end{aligned}
$$

Here, $q$ and $p$ are the photon and nucleon four-momenta (cf. Fig. 1), $\nu$ is the photon lab-frame energy, $Q^{2}=-q^{2}$ is the photon virtuality, and $\gamma^{\mu \nu}=\frac{1}{2}\left[\gamma^{\mu}, \gamma^{\nu}\right]$ and $\gamma^{\mu \nu \alpha}=$ $\frac{1}{2}\left(\gamma^{\mu} \gamma^{\nu} \gamma^{\alpha}-\gamma^{\alpha} \gamma^{\nu} \gamma^{\mu}\right)$ are the usual Dirac matrices. Alternatively, one can use the following laboratory-frame amplitudes:

$$
\begin{gathered}
g_{T T}\left(\nu, Q^{2}\right)=\frac{\nu}{M_{N}}\left[S_{1}\left(\nu, Q^{2}\right)-\frac{Q^{2}}{M_{N} \nu} S_{2}\left(\nu, Q^{2}\right)\right], \\
g_{L T}\left(\nu, Q^{2}\right)=\frac{Q}{M_{N}}\left[S_{1}\left(\nu, Q^{2}\right)+\frac{\nu}{M_{N}} S_{2}\left(\nu, Q^{2}\right)\right],
\end{gathered}
$$

introduced in Eq. (A2). The optical theorem relates the absorptive parts of the forward VVCS amplitudes to the nucleon structure functions or the cross sections of virtual photoabsorption:

$$
\begin{aligned}
\operatorname{Im} S_{1}\left(\nu, Q^{2}\right) & =\frac{4 \pi^{2} \alpha}{\nu} g_{1}\left(x, Q^{2}\right) \\
& =\frac{M_{N} \nu K\left(\nu, Q^{2}\right)}{\nu^{2}+Q^{2}}\left[\frac{Q}{\nu} \sigma_{L T}\left(\nu, Q^{2}\right)+\sigma_{T T}\left(\nu, Q^{2}\right)\right] \\
\operatorname{Im} S_{2}\left(\nu, Q^{2}\right) & =\frac{4 \pi^{2} \alpha M_{N}}{\nu^{2}} g_{2}\left(x, Q^{2}\right) \\
& =\frac{M_{N}^{2} K\left(\nu, Q^{2}\right)}{\nu^{2}+Q^{2}}\left[\frac{\nu}{Q} \sigma_{L T}\left(\nu, Q^{2}\right)-\sigma_{T T}\left(\nu, Q^{2}\right)\right]
\end{aligned}
$$

with $\alpha$ the fine structure constant and $K\left(\nu, Q^{2}\right)$ the photon flux factor. Note that the photon flux factor in the optical theorem and the cross sections, measured in electroproduction processes, is a matter of convention and has to be chosen in both quantities consistently. In what follows, we use Gilman's flux factor: 


$$
K\left(\nu, Q^{2}\right) \equiv|\vec{q}|=\sqrt{\nu^{2}+Q^{2}}
$$

The helicity-difference photoabsorption cross section is defined as $\sigma_{T T}=1 / 2\left(\sigma_{1 / 2}-\sigma_{3 / 2}\right)$, where the photons are transversely polarized, and the subscripts on the right-hand side indicate the total helicities of the $\gamma^{*} N$ states. The cross section $\sigma_{L T}$ corresponds to a simultaneous helicity change of the photon and nucleon spin flip, such that the total helicity is conserved. A detailed derivation of the connection between this response function and the photoabsorption cross sections can be found in Appendix B. The forward VVCS amplitudes satisfy dispersion relations derived from the general principles of analyticity and causality ${ }^{1}$ :

$$
\begin{aligned}
S_{1}\left(\nu, Q^{2}\right) & =\frac{16 \pi \alpha M_{N}}{Q^{2}} \int_{0}^{1} \mathrm{~d} x \frac{g_{1}\left(x, Q^{2}\right)}{1-x^{2}\left(\nu / \nu_{\mathrm{el}}\right)^{2}-i 0^{+}} \\
& =\frac{2 M_{N}}{\pi} \int_{\nu_{\mathrm{el}}}^{\infty} \mathrm{d} \nu^{\prime} \frac{\nu^{\prime 2}\left[\frac{Q}{\nu^{\prime}} \sigma_{L T}+\sigma_{T T}\right]\left(\nu^{\prime}, Q^{2}\right)}{\sqrt{\nu^{\prime 2}+Q^{2}}\left(\nu^{\prime 2}-\nu^{2}-i 0^{+}\right)} \\
\nu S_{2}\left(\nu, Q^{2}\right) & =\frac{16 \pi \alpha M_{N}^{2}}{Q^{2}} \int_{0}^{1} \mathrm{~d} x \frac{g_{2}\left(x, Q^{2}\right)}{1-x^{2}\left(\nu / \nu_{\mathrm{el}}\right)^{2}-i 0^{+}} \\
& =\frac{2 M_{N}^{2}}{\pi} \int_{\nu_{\mathrm{el}}}^{\infty} \mathrm{d} \nu^{\prime} \frac{\nu^{\prime 2}\left[\frac{\nu^{\prime}}{Q} \sigma_{L T}-\sigma_{T T}\right]\left(\nu^{\prime}, Q^{2}\right)}{\sqrt{\nu^{\prime 2}+Q^{2}}\left(\nu^{\prime 2}-\nu^{2}-i 0^{+}\right)}
\end{aligned}
$$

with $\nu_{\mathrm{el}}=Q^{2} / 2 M_{N}$ the elastic threshold.

\section{B. Low-energy expansions and relations to polarizabilities}

The VVCS amplitudes naturally split into nucleon-pole $\left(S_{i}^{\text {pole }}\right)$ and nonpole $\left(S_{i}^{\text {nonpole }}\right)$ parts, or Born $\left(S_{i}^{\text {Born }}\right)$ and nonBorn $\left(\bar{S}_{i}\right)$ parts:

$$
S_{i}=S_{i}^{\text {pole }}+S_{i}^{\text {nonpole }}=S_{i}^{\text {Born }}+\bar{S}_{i}
$$

The Born amplitudes are given uniquely in terms of the nucleon form factors [1]:

$$
\begin{aligned}
& S_{1}^{\mathrm{Born}}\left(\nu, Q^{2}\right)=\frac{2 \pi \alpha}{M_{N}}\left\{\frac{Q^{2} G_{M}\left(Q^{2}\right) F_{1}\left(Q^{2}\right)}{\nu_{\mathrm{el}}^{2}-\nu^{2}}-F_{2}^{2}\left(Q^{2}\right)\right\}, \\
& S_{2}^{\mathrm{Born}}\left(\nu, Q^{2}\right)=-\frac{2 \pi \alpha \nu}{\nu_{\mathrm{el}}^{2}-\nu^{2}} G_{M}\left(Q^{2}\right) F_{2}\left(Q^{2}\right) .
\end{aligned}
$$

The same is true for the nucleon-pole amplitudes, which are related to the Born amplitudes in the following way:

\footnotetext{
${ }^{1}$ The dispersion relation for $\nu S_{2}\left(\nu, Q^{2}\right)$ is used because it is pole-free in the limit $Q^{2} \rightarrow 0$, and then $\nu \rightarrow 0$ [cf. Eq. (7b)].
}

$$
\begin{aligned}
& S_{1}^{\text {pole }}\left(\nu, Q^{2}\right)=S_{1}^{\text {Born }}\left(\nu, Q^{2}\right)+\frac{2 \pi \alpha}{M_{N}} F_{2}^{2}\left(Q^{2}\right), \\
& S_{2}^{\text {pole }}\left(\nu, Q^{2}\right)=S_{2}^{\text {Born }}\left(\nu, Q^{2}\right) .
\end{aligned}
$$

Here, we used the elastic Dirac and Pauli form factors $F_{1}\left(Q^{2}\right)$ and $F_{2}\left(Q^{2}\right)$, related to the electric and magnetic Sachs form factors $G_{E}\left(Q^{2}\right)$ and $G_{M}\left(Q^{2}\right)$ through

$$
\begin{gathered}
F_{1}\left(Q^{2}\right)=\frac{G_{E}\left(Q^{2}\right)+\tau G_{M}\left(Q^{2}\right)}{1+\tau}, \\
F_{2}\left(Q^{2}\right)=\frac{G_{M}\left(Q^{2}\right)-G_{E}\left(Q^{2}\right)}{1+\tau},
\end{gathered}
$$

where $\tau=Q^{2} / 4 M_{N}^{2}$.

A LEX of Eq. (5), in combination with the unitarity relations given in Eq. (3), establishes various sum rules relating the nucleon properties (electromagnetic moments, polarizabilities) to experimentally observable response functions $[1,3]$. The leading terms in the LEX of the real Compton scattering (RCS) amplitudes are determined uniquely by charge, mass, and anomalous magnetic moment, as the global properties of the nucleon. These lowest-order terms represent the celebrated low-energy theorem (LET) of Low, Gell-Mann, and Goldberger $[39,40]$. The polarizabilities, related to the internal structure of the nucleon, enter the LEX at higher orders. They make up the non-Born amplitudes and can be related to moments of inelastic structure functions.

The process of VVCS can be realized experimentally in electron-nucleon scattering, where a virtual photon is exchanged between the electron and the nucleon. This virtual photon acts as a probe whose resolution depends on its virtuality $Q^{2}$. In this way, one can access the so-called generalized polarizabilities, which extend the notion of polarizabilities to the case of response to finite momentum transfer. The generalized forward spin polarizability $\gamma_{0}\left(Q^{2}\right)$ and the longitudinal-transverse polarizability $\delta_{L T}\left(Q^{2}\right)$ are most naturally defined via the LEX of the non-Born part of the lab-frame VVCS amplitudes [1]:

$$
\begin{aligned}
\frac{1}{4 \pi} g_{T T}^{\text {nonpole }}\left(\nu, Q^{2}\right)= & \frac{2 \alpha}{M_{N}^{2}} I_{A}\left(Q^{2}\right) \nu+\gamma_{0}\left(Q^{2}\right) \nu^{3} \\
& +\bar{\gamma}_{0}\left(Q^{2}\right) \nu^{5}+\cdots, \\
\frac{1}{4 \pi} g_{L T}^{\text {nonpole }}\left(\nu, Q^{2}\right)= & \frac{2 \alpha}{M_{N}^{2}} I_{3}\left(Q^{2}\right) Q+\delta_{L T}\left(Q^{2}\right) \nu^{2} Q+\cdots
\end{aligned}
$$

Their definitions in terms of integrals over structure functions are postponed to Eqs. (19) and (22). Here, we only give the definition of the moment $I_{3}\left(Q^{2}\right)$ : 


$$
\begin{aligned}
I_{3}\left(Q^{2}\right) & =\frac{M_{N}^{2}}{4 \pi^{2} \alpha} \int_{\nu_{0}}^{\infty} \mathrm{d} \nu \frac{K\left(\nu, Q^{2}\right)}{\nu Q} \sigma_{L T}\left(\nu, Q^{2}\right) \\
& =\frac{2 M_{N}^{2}}{Q^{2}} \int_{0}^{x_{0}} \mathrm{~d} x\left[g_{1}\left(x, Q^{2}\right)+g_{2}\left(x, Q^{2}\right)\right],
\end{aligned}
$$

which is related to the Schwinger sum rule in the real photon limit [7-9,41,42]. The LEX of the nonpole part of the covariant VVCS amplitudes can be described entirely in terms of moments of inelastic spin structure functions [up to $\mathcal{O}\left(\nu^{4}\right)[43]$ ]:

$$
\begin{aligned}
\frac{1}{4 \pi} S_{1}^{\text {nonpole }}\left(\nu, Q^{2}\right)= & \frac{2 \alpha}{M_{N}} I_{1}\left(Q^{2}\right)+\left\{\frac { 2 \alpha } { M _ { N } Q ^ { 2 } } \left[I_{A}\left(Q^{2}\right)\right.\right. \\
& \left.\left.-I_{1}\left(Q^{2}\right)\right]+M_{N} \delta_{L T}\left(Q^{2}\right)\right\} \nu^{2}, \quad(12 \mathrm{a}) \\
\frac{1}{4 \pi} \nu S_{2}^{\text {nonpole }}\left(\nu, Q^{2}\right)= & 2 \alpha I_{2}\left(Q^{2}\right)+\frac{2 \alpha}{Q^{2}}\left[I_{1}\left(Q^{2}\right)-I_{A}\left(Q^{2}\right)\right] \nu^{2} .
\end{aligned}
$$

$I_{1}\left(Q^{2}\right)$ and $I_{A}\left(Q^{2}\right)$ are generalizations of the famous Gerasimov-Drell-Hearn (GDH) sum rule [5,6] from RCS to the case of virtual photons [1]. Their definitions are given in Eqs. (26) and (32). $I_{2}\left(Q^{2}\right)$ is the well-known BurkhardtCottingham (BC) sum rule [44],

$I_{2}\left(Q^{2}\right) \equiv \frac{2 M_{N}^{2}}{Q^{2}} \int_{0}^{x_{0}} \mathrm{~d} x g_{2}\left(x, Q^{2}\right)=\frac{1}{4} F_{2}\left(Q^{2}\right) G_{M}\left(Q^{2}\right)$,

which can be written as a "superconvergence sum rule":

$$
\frac{Q^{2}}{16 \pi \alpha M_{N}^{2}} \lim _{\nu \rightarrow 0} \nu S_{2}\left(\nu, Q^{2}\right)=\int_{0}^{1} \mathrm{~d} x g_{2}\left(x, Q^{2}\right)=0 .
$$

The latter is valid for any value of $Q^{2}$ provided that the integral converges for $x \rightarrow 0$. Combining Eq. (5) with the above LEXs of the VVCS amplitudes, we can relate $I_{A}\left(Q^{2}\right)$, $I_{1}\left(Q^{2}\right), \gamma_{0}\left(Q^{2}\right)$, and $\delta_{L T}\left(Q^{2}\right)$ to moments of inelastic structure functions (see Sec. III). It is important to note that only $\gamma_{0}\left(Q^{2}\right)$ and $\delta_{L T}\left(Q^{2}\right)$ are generalized polarizabilities. The relation of the inelastic moments $I_{A}\left(Q^{2}\right)$ and $I_{1}\left(Q^{2}\right)$ to polarizabilities will be discussed in detail in Secs. III C and III D. The difference between $\bar{S}_{1}\left(\nu, Q^{2}\right)$ and $S_{1}^{\text {nonpole }}\left(\nu, Q^{2}\right)$ [cf. Eq. (8)] will be important in this context.

\section{Details on $\chi$ PT calculation and uncertainty estimate}

In this work, we calculated the next-to-leading-order (NLO) prediction of $\mathrm{B} \chi \mathrm{PT}$ for the polarized non-Born VVCS amplitudes. This includes the leading pion-nucleon $(\pi N)$ loops (see Ref. [32][Fig. 1]), as well as the subleading tree-level Delta exchange ( $\Delta$ exchange) (see Ref. [30]
[Fig. 2]) and the pion-Delta $(\pi \Delta)$ loops (see Ref. [32] [Fig. 2]). In the $\delta$-power-counting scheme [36], the LO and NLO non-Born VVCS amplitudes and polarizabilities are of $\mathcal{O}\left(p^{3}\right)$ and $\mathcal{O}\left(p^{4} / \Delta\right)$, respectively. ${ }^{2}$ The low-energy constants (LECs) are listed in Table I, sorted by the order at which they appear in our calculation. At the given orders, there are no "new" LECs that would need to be fitted from Compton processes. For more details on the $\mathrm{B} \chi \mathrm{PT}$ formalism, we refer to Ref. [30], where power counting, predictive orders (Sec. III A), and the renormalization procedure (Sec. III B) are discussed.

A few remarks are in order for the inclusion of the $\Delta(1232)$ and the tree-level $\Delta$-exchange contribution. In contrast to Ref. [32], we include the Coulomb-quadrupole (C2) $N \rightarrow \Delta$ transition described by the LEC $g_{C}$. The relevant Lagrangian describing the nonminimal $\gamma^{*} N \Delta$ coupling $[33,34]$ (note that in these references the overall sign of $g_{C}$ is inconsistent between the Lagrangian and Feynman rules) reads

$$
\begin{aligned}
\mathcal{L}_{\Delta}^{(2)}= & \frac{3 e}{2 M_{N} M_{+}} \bar{N} T_{3}\left\{i g_{M} \tilde{F}^{\mu \nu} \partial_{\mu} \Delta_{\nu}-g_{E} \gamma_{5} F^{\mu \nu} \partial_{\mu} \Delta_{\nu}\right. \\
& \left.+i \frac{g_{C}}{M_{\Delta}} \gamma_{5} \gamma^{\alpha}\left(\partial_{\alpha} \Delta_{\nu}-\partial_{\nu} \Delta_{\alpha}\right) \partial_{\mu} F^{\mu \nu}\right\}+ \text { H.c. },
\end{aligned}
$$

with $M_{+}=M_{N}+M_{\Delta}$ and the dual of the electromagnetic field strength tensor $\tilde{F}^{\mu \nu}=\frac{1}{2} \epsilon^{\mu \nu \rho \lambda} F_{\rho \lambda}$. Even though the Coulomb coupling is subleading compared with the electric and magnetic couplings $\left(g_{E}\right.$ and $\left.g_{M}\right)$, its relatively large magnitude (cf. Table I) makes it numerically important, for instance, in $\gamma_{0}\left(Q^{2}\right)$. Furthermore, we study the effect of modifying the magnetic coupling using a dipole form factor,

$$
g_{M} \rightarrow \frac{g_{M}}{\left[1+(Q / \Lambda)^{2}\right]^{2}},
$$

where $\Lambda^{2}=0.71 \mathrm{GeV}^{2}$. The inclusion of this $Q^{2}$ dependence mimics the form expected from vector-meson dominance. It is motivated by observing the importance of this form factor for the correct description of the electroproduction data [33].

To estimate the uncertainties of our NLO predictions, we define

$$
\tilde{\delta}\left(Q^{2}\right)=\sqrt{\left(\frac{\Delta}{M_{N}}\right)^{2}+\left(\frac{Q^{2}}{2 M_{N} \Delta}\right)^{2}},
$$

such that the neglected next-to-next-to-leading order terms are expected to be of relative size $\tilde{\delta}^{2}$ [33]. The uncertainties

\footnotetext{
${ }^{2}$ In the full Compton amplitude, there is a lower order contribution coming from the Born terms, leading to a shift in nomenclature by one order: the LO contribution referred to as the NLO contribution, etc. (See, e.g., Ref. [45].)
} 

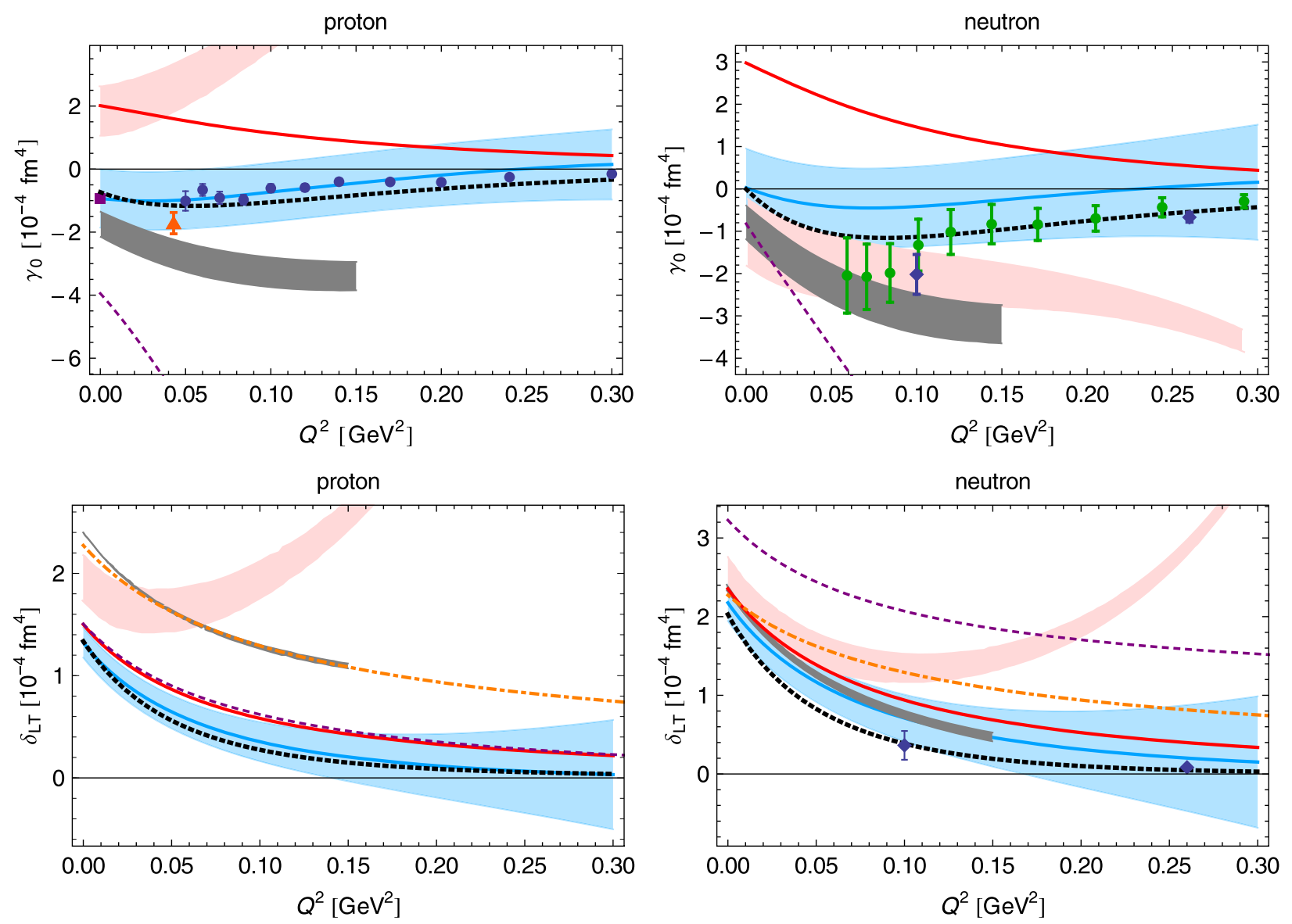

FIG. 2. Upper panel: Generalized forward spin polarizability for the proton (left) and neutron (right) as a function of $Q^{2}$. The result of this work, the NLO B $\chi$ PT prediction, is shown by the blue solid line and the blue band. The red line represents the LO B $\chi$ PT result. The purple short-dashed line is the $\mathcal{O}\left(p^{4}\right)$ HB result from Ref. [51]. The black dotted line is the MAID model prediction [61-63], which is taken from Ref. [1] (proton) and Ref. [20] (neutron). The pink band is the IR $+\Delta$ result from Ref. [60], and the gray band is the $\mathrm{B} \chi \mathrm{PT}+\Delta$ result from Ref. [31]. Empirical extractions for the proton: Ref. [18] (blue dots), Ref. [64] (purple square), and Ref. [57] (orange triangle; uncertainties added in quadrature); and neutron: Ref. [20] (blue diamonds) and Ref. [58] (green dots; statistical and systematic uncertainties added in quadrature). Lower Panel: Longitudinal-transverse spin polarizability for the proton (left) and neutron (right). The orange dot-dashed and purple short-dashed lines are the $\mathcal{O}\left(p^{3}\right)$ and $\mathcal{O}\left(p^{4}\right) \mathrm{HB}$ results from Ref. [51]. The pink band is the IR result from Ref. [60] and the gray band is the covariant $\mathrm{B} \chi \mathrm{PT}+\Delta$ result from Ref. [31]. The black dotted line is the MAID model prediction [61-63]; note that for the proton we use the updated estimate from Ref. [1] obtained using the $\pi, \eta, \pi \pi$ channels.

in the values of the parameters in Table I have a much smaller impact compared to the truncation uncertainty and can be neglected. Unfortunately, $\Delta I_{A}\left(Q^{2}\right), \gamma_{0}\left(Q^{2}\right)$, and $\bar{\gamma}_{0}\left(Q^{2}\right)$, i.e., the sum rules involving the cross section $\sigma_{T T}\left(\nu, Q^{2}\right)$, as well as the polarizability $\Delta I_{1}\left(Q^{2}\right)$, turn out

TABLE I. Parameters (fundamental and low-energy constants) [46] at the order they appear first. The $\pi N \Delta$ coupling constant $h_{A}$ is fit to the experimental Delta width and the $\gamma^{*} N \Delta$ coupling constants $g_{M}, g_{E}$, and $g_{C}$ are taken from the pion photoproduction study of Ref. [33].

\begin{tabular}{ll}
\hline \hline $\mathcal{O}\left(p^{2}\right)$ & $\alpha=1 /(137.04), M_{N}=M_{p}=938.27 \mathrm{MeV}$ \\
$\mathcal{O}\left(p^{3}\right)$ & $g_{A}=1.270, f_{\pi}=92.21 \mathrm{MeV}, m_{\pi}=139.57 \mathrm{MeV}$ \\
$\mathcal{O}\left(p^{4} / \Delta\right)$ & $M_{\Delta}=1232 \mathrm{MeV}, h_{A} \equiv 2 g_{\pi N \Delta}=2.85$ \\
& $g_{M}=2.97, g_{E}=-1.0, g_{C}=-2.6$ \\
\hline \hline
\end{tabular}

to be numerically small. Their smallness suggests a cancellation of leading orders (which can indeed be confirmed by looking at separate contributions as shown below). Therefore, an error of $\tilde{\delta}^{2}\left(Q^{2}\right) P\left(Q^{2}\right)$, where $P\left(Q^{2}\right)$ is a generalized polarizability, might underestimate the theoretical uncertainty for some of the NLO predictions. To avoid this, we estimate the uncertainty of our NLO polarizability predictions by

$$
\begin{aligned}
\sigma & P\left(Q^{2}\right) \\
= & \left\{\operatorname{Max}\left[\tilde{\delta}^{4}(0) P(0)^{2}, \tilde{\delta}^{4}(0) P^{\mathrm{LO}}(0)^{2}, \tilde{\delta}^{2}(0) P^{\mathrm{NLO}}(0)^{2}\right]\right. \\
& +\operatorname{Max}\left[\tilde{\delta}^{4}\left(Q^{2}\right)\left[P\left(Q^{2}\right)-P(0)\right]^{2}\right. \\
& \tilde{\delta}^{4}\left(Q^{2}\right)\left[P^{\mathrm{LO}}\left(Q^{2}\right)-P^{\mathrm{LO}}(0)\right]^{2} \\
& \left.\left.\tilde{\delta}^{2}\left(Q^{2}\right)\left[P^{\mathrm{NLO}}\left(Q^{2}\right)-P^{\mathrm{NLO}}(0)\right]^{2}\right]\right\}^{1 / 2}
\end{aligned}
$$


where $P^{\mathrm{LO}}\left(Q^{2}\right)$ is the $\pi N$-loop contribution, $P^{\mathrm{NLO}}\left(Q^{2}\right)$ are the $\Delta$-exchange and $\pi \Delta$-loop contributions, and $P\left(Q^{2}\right)=P^{\mathrm{LO}}\left(Q^{2}\right)+P^{\mathrm{NLO}}\left(Q^{2}\right)$. This error prescription is similar to the one used in, e.g., Refs. [47-50]. Here, since we are interested in the generalized polarizabilities, we added in quadrature the error due to the real-photon piece $P(0)$ and the $Q^{2}$-dependent remainder $P\left(Q^{2}\right)-P(0)$. Note that $I_{A}(0)$ and $I_{1}(0)$ are given by the elastic Pauli form factor, which is not part of our $\mathrm{B} \chi \mathrm{PT}$ prediction and is considered to be exact.

Note that our result for the spin polarizabilities (and the unpolarized moments [30]) are NLO predictions only at low momentum transfers $Q \simeq m_{\pi}$. At larger values of $Q \gtrsim \Delta$, they become incomplete LO predictions. Indeed, in this regime the $\Delta$ propagators do not carry additional suppression compared to the nucleon propagators, and the $\pi \Delta$ loops are promoted to LO. In general, we only expect a rather small contribution from omitted $\pi \Delta$ loops to the $Q^{2}$ dependence of the polarizabilities, since $\pi \Delta$ loops show rather weak dependence on $Q^{2}$ compared with the $\Delta$ exchange or $\pi N$ loops. Nevertheless, this issue has to be reflected in the error estimate. Since the polarizabilities at the real-photon point $P(0)$ are not affected, it is natural to separate the error on the $Q^{2}$-dependent remainder $P\left(Q^{2}\right)-P(0)$, as done in Eq. (18). To accommodate for the potential loss of precision above $Q \gtrsim \Delta$, we define the relative error $\tilde{\delta}\left(Q^{2}\right)$ as growing with increasing $Q^{2}$ [see Eq. (17)].

Upon expanding our results in powers of the inverse nucleon mass, $M_{N}^{-1}$, we are able to reproduce existing results of heavy-baryon $\chi \mathrm{PT}(\mathrm{HB} \chi \mathrm{PT})$ at LO. We, however, do not see a rationale to drop the higher-order $M_{N}^{-1}$ terms when they are not negligible (i.e., when their actual size exceeds by far the natural estimate for the size of higher-order terms). Comparing our $\mathrm{B} \chi \mathrm{PT}$ predictions to $\mathrm{HB} \chi \mathrm{PT}$, we will also see a deficiency of $\mathrm{HB} \chi \mathrm{PT}$ in the description of the $Q^{2}$ behavior of the polarizabilities. Note that the $\mathcal{O}\left(p^{4}\right) \mathrm{HB} \chi \mathrm{PT}$ results from Refs. [51,52], which we use here for comparison, do not include the $\Delta$. These references studied the leading effect of the latter in the $\mathrm{HB} \chi \mathrm{PT}$ framework, using the small-scale expansion [35], observing no qualitative improvement in the $\mathrm{HB} \chi \mathrm{PT}$ description of the empirical data [51,52] when including it. We therefore choose to use the $\mathcal{O}\left(p^{4}\right)$ results as the representative $\mathrm{HB} \chi \mathrm{PT}$ curves.

Another approach used in the literature to calculate the polarizabilities in $\chi \mathrm{PT}$ is the infrared regularization (IR) scheme, introduced in Ref. [53]. This covariant approach tries to solve the power counting violation observed in Ref. [54] by dropping the regular parts of the loop integrals that contain the power-counting-breaking terms. However, this subtraction scheme modifies the analytic structure of the loop contributions and may lead to unexpected problems, as was shown in Ref. [55]. As we will see in the next section, the IR approach also fails to describe the $Q^{2}$ behavior of the polarizabilities.

\section{RESULTS AND DISCUSSION}

We now present the NLO $\mathrm{B} \chi \mathrm{PT}$ predictions for the nucleon polarizabilities and selected moments of the nucleon spin structure functions. Our results are obtained from the calculated non-Born VVCS amplitudes and the LEXs in Eqs. (10) and (12). For a cross-check, we used the photoabsorption cross sections described in Appendix B. In addition to the full NLO results, we also analyze the individual contributions from the $\pi N$ loops, the $\Delta$ exchange, and the $\pi \Delta$ loops.

\section{A. $\gamma_{0}\left(Q^{2}\right)$-generalized forward spin polarizability}

The forward spin polarizability,

$$
\begin{aligned}
\gamma_{0}\left(Q^{2}\right) & =\frac{1}{2 \pi^{2}} \int_{\nu_{0}}^{\infty} \mathrm{d} \nu \sqrt{1+\frac{Q^{2}}{\nu^{2}}} \frac{\sigma_{T T}\left(\nu, Q^{2}\right)}{\nu^{3}} \\
& =\frac{16 \alpha M_{N}^{2}}{Q^{6}} \int_{0}^{x_{0}} \mathrm{~d} x x^{2}\left[g_{1}\left(x, Q^{2}\right)-\frac{4 M_{N}^{2} x^{2}}{Q^{2}} g_{2}\left(x, Q^{2}\right)\right]
\end{aligned}
$$

provides information about the spin-dependent response of the nucleon to transversal photon probes. The RCS analogue of the above generalized forward spin polarizability sum rule is sometimes referred to as the GellMann, Goldberger, and Thirring (GGT) sum rule [11]. At $Q^{2}=0$, the forward spin polarizability is expressed through the lowest-order spin polarizabilities of RCS as $\gamma_{0}=-\left(\gamma_{E 1 E 1}+\gamma_{M 1 M 1}+\gamma_{E 1 M 2}+\gamma_{M 1 E 2}\right)$. The forward spin polarizability of the proton is relevant for an accurate knowledge of the (muonic-)hydrogen hyperfine splitting, as it controls the leading proton-polarizability correction $[16,56]$.

The $\pi N$-loop, $\Delta$-exchange, and $\pi \Delta$-loop contributions to the NLO B $\chi$ PT prediction of the forward spin polarizability amount to, in units of $10^{-4} \mathrm{fm}^{4}$,

$$
\begin{aligned}
& \gamma_{0 p}=-0.93(92) \approx 2.01-2.84-0.10, \\
& \gamma_{0 n}=0.03(92) \approx 2.98-2.84-0.10,
\end{aligned}
$$

while the slope is composed as follows, in units of $10^{-4} \mathrm{fm}^{6}$ :

$\left.\frac{\mathrm{d} \gamma_{0 p}\left(Q^{2}\right)}{\mathrm{d} Q^{2}}\right|_{Q^{2}=0}=-0.22(4) \approx-0.33+0.11+0.01$,

$\left.\frac{\mathrm{d} \gamma_{0 n}\left(Q^{2}\right)}{\mathrm{d} Q^{2}}\right|_{Q^{2}=0}=-0.61(7) \approx-0.73+0.11+0.01$.

Figure 2 (upper panel) shows our NLO prediction, as well as the LO $\pi N$ loops, compared to different experimental and theoretical results. For the proton, we have one determination at the real-photon point by the GDH 
Collaboration [19], $\gamma_{0 p}=-1.00(8)(12) \times 10^{-4} \mathrm{fm}^{4}$, and further Jefferson Laboratory data [18,57] at very low $Q^{2}$. For the neutron, only data at finite $Q^{2}$ are available [20,58]. The experimental data for the proton are fairly well reproduced in the whole $Q^{2}$ range considered here, while for the neutron the agreement improves with increasing $Q^{2}$. The HB limit of our $\pi N$-loop contribution reproduces the results published in Refs. [51,59] for arbitrary $Q^{2}$. In addition, our prediction is compared to the MAID model $[1,20]$, the IR $+\Delta$ calculation of Ref. [60], and the $\mathrm{B} \chi \mathrm{PT}+\Delta$ result of Ref. [31].

The $\pi N$-production channel gives a positive contribution to the photoabsorption cross section $\sigma_{T T}\left(\nu, Q^{2}\right)$ at low $Q^{2}$ (cf. Fig. 10). Accordingly, one observes that the $\pi N$ loops give a sizable positive contribution to $\gamma_{0}\left(Q^{2}\right)$. The Delta, on the other hand, has a very large effect by canceling the $\pi N$ loops and bringing the result close to the empirical data. From Fig. 3 (upper panel), one can see that it is the $\Delta$ exchange what dominates, while $\pi \Delta$ loops are negligible. This was expected, since the forward spin polarizability sum rule is an integral over the helicity-difference cross section, in which $\sigma_{3 / 2}$ is governed by the Delta at low energies (the relevant energy region for the sum rule).

To elucidate the difference between the present calculation and the one from Ref. [31], we note that the two calculations differ in the following important aspects. First, Ref. [31] uses the small-scale counting [65] that considers $\Delta$ and $m_{\pi}$ as being of the same size, $\Delta \sim m_{\pi}$. In practice, this results in a set of $\pi \Delta$-loop graphs which contains graphs with one or two $\gamma \Delta \Delta$ couplings and hence two or three Delta propagators. Such graphs are suppressed in the $\delta$ counting and thus omitted from our calculation while present in that of Ref. [31]. Second, the Lagrangians describing the interaction of the Delta are constructed differently and assume slightly different values for the coupling constants. In particular, we employ (where possible) the so-called "consistent" couplings to the Delta field, i.e., those couplings that project out the spurious degree of freedom (see Refs. [37,66,67]). The authors of Ref. [31], on the other hand, use couplings where the consistency in this sense is not enforced. The effects of these differences are of higher order in the $\delta$-counting
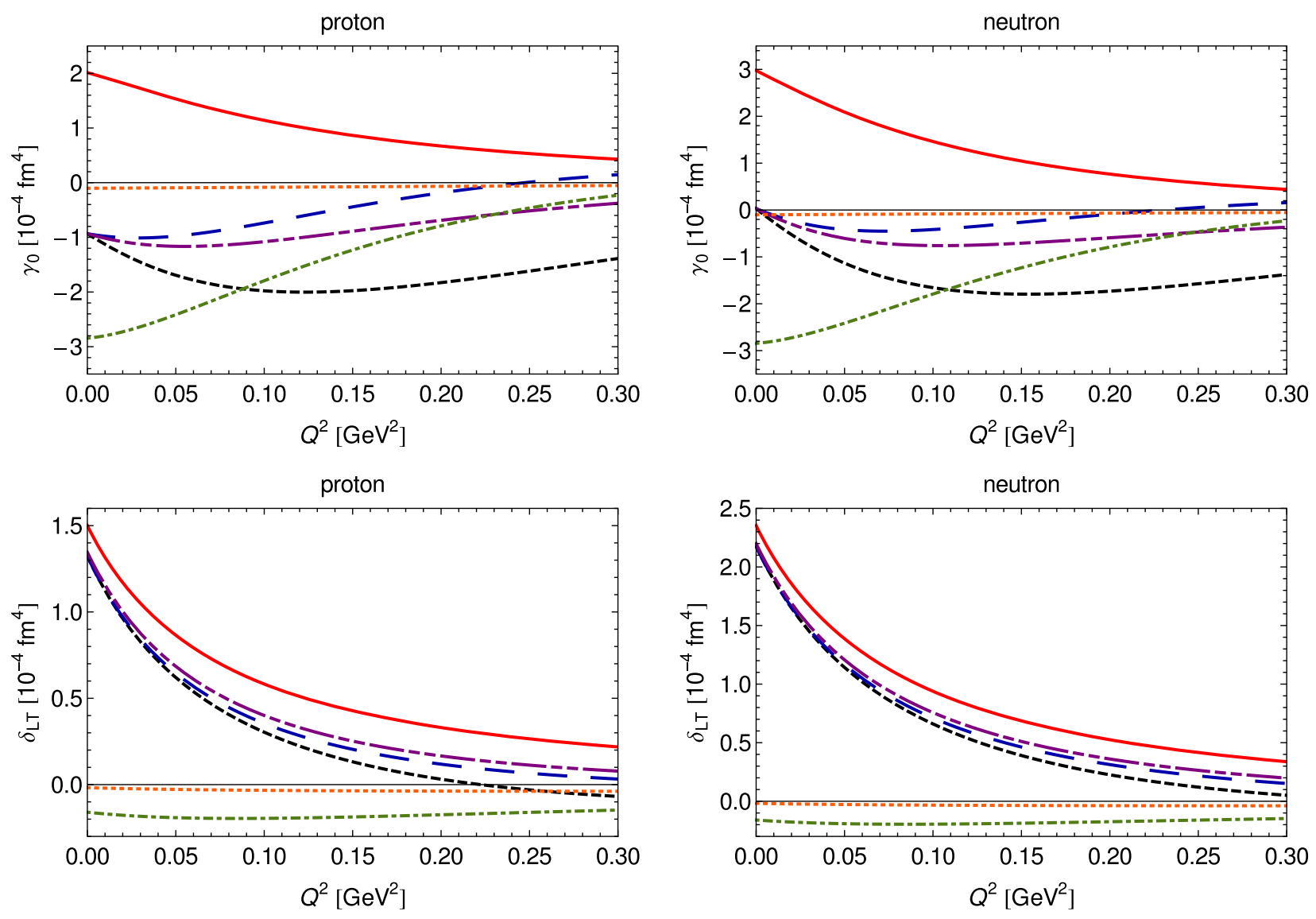

FIG. 3. Contributions of the different orders to the chiral predictions of $\gamma_{0}\left(Q^{2}\right)$ (upper panel) and $\delta_{L T}\left(Q^{2}\right)$ (lower panel) for the proton (left) and neutron (right). Red solid line: $\pi N$-loop contribution; green dot-dashed line: $\Delta$-exchange contribution; orange dotted line: $\pi \Delta$-loop contribution; blue long-dashed line: total result; purple dot-dot-dashed line: total result without $g_{C}$ contribution; and black short-dashed line: total result without $g_{M}$ dipole. 
expansion, and their contribution to the $Q^{2}$ dependence of the considered polarizabilities is expected to be rather small; however, the differences at $Q^{2}=0$ could be noticeable [68].

Finally, as mentioned in Sec. II C, we are including a dipole form factor in the magnetic coupling $g_{M}$, a modification absent in Ref. [31]. This modification is expected to be needed in order to generate the correct $Q^{2}$ behavior of the polarizabilities that receive a significant contribution from the magnetic $\gamma^{*} N \Delta$ transition, such as $\gamma_{0}\left(Q^{2}\right)$. Figure 2 (upper panel) shows that our predictions for the $Q^{2}$ dependence of $\gamma_{0}\left(Q^{2}\right)$ differ quite significantly from those of Ref. [31]. The main reason for this is the dipole form factor that indeed drives the curves closer to the experimental data. Another polarizability that shows a similar importance of the dipole form factor is the closely related $I_{A}\left(Q^{2}\right)$, considered below in Sec. III C, and shown in Fig. 4 (upper panel). In other polarizabilities, such as $\delta_{L T}\left(Q^{2}\right)$ shown in Fig. 2 (lower panel), the magnetic transition is not so prominent, and so is the effect of the dipole form factor on the $Q^{2}$ dependence. The effect of the form factor on the polarizabilities is further illustrated in Figs. 3, 5, and 9, where one can see the total result with the $g_{M}$ dipole compared to the total result without it.

Concerning the generalized forward spin polarizability, the experimental data for $\gamma_{0 n}\left(Q^{2}\right)$ at very low $Q^{2}$ slightly favor the $\mathrm{B} \chi \mathrm{PT}$ prediction without inclusion of the dipole form factor [31]. In general, our $\mathrm{B} \chi \mathrm{PT}$ prediction is able to describe all experimental data within errors and shows perfect agreement for $\gamma_{0 p}\left(Q^{2}\right)$ at $Q^{2}<0.3 \mathrm{GeV}^{2}$
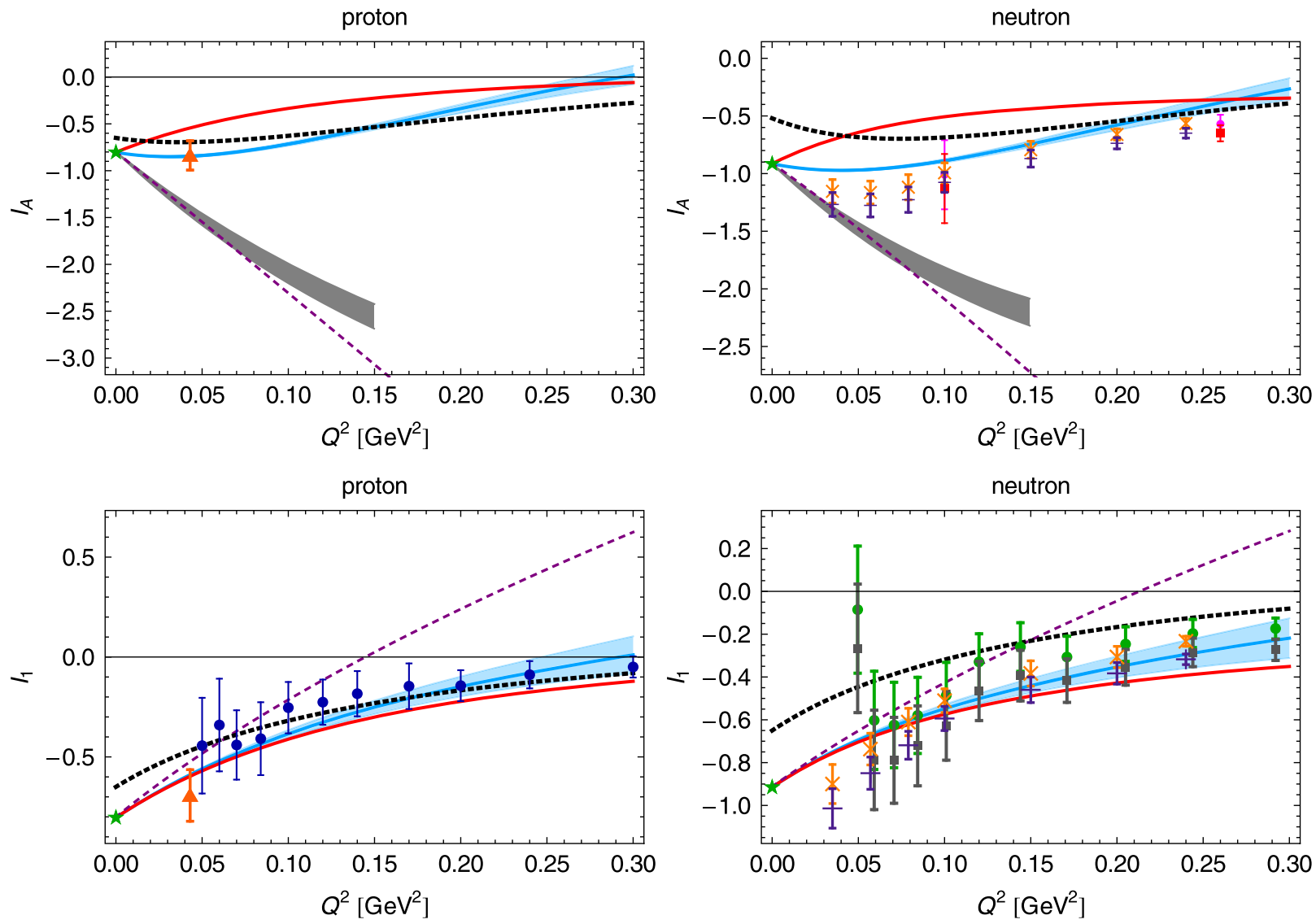

FIG. 4. Upper panel: The generalized GDH integral $I_{A}\left(Q^{2}\right)$ for the proton (left) and neutron (right) as a function of $Q^{2}$. The result of this work, the NLO B $\chi$ PT prediction, is shown by the blue solid line and the blue band. The red line represents the LO B $\chi$ PT result. The purple short-dashed line is the $\mathcal{O}\left(p^{4}\right)$ HB result from Refs. [51,52]. The gray band is the $\mathrm{B} \chi \mathrm{PT}+\Delta$ result from Ref. [31]. The black dotted line is the MAID model prediction [69]. Experimental extractions for the proton: Ref. [57] (orange triangle; uncertainties added in quadrature); and neutron: Refs. [21]/[27], where magenta dots/orange diagonal crosses correspond to data and red squares/lilac crosses correspond to data plus extrapolation to unmeasured energy regions. The green stars at the real-photon point are derived from the anomalous magnetic moments: $\varkappa_{p} \approx 1.793$ and $\varkappa_{n} \approx-1.913$ [70]. Lower panel: The generalized GDH integral $I_{1}\left(Q^{2}\right)$ for the proton (left) and neutron (right) as a function of $Q^{2}$. The purple short-dashed line is the HB result from Ref. [52]. Experimental extractions for the proton: Ref. [18] (blue dots) and Ref. [57] (orange triangle; uncertainties added in quadrature); and neutron: Refs. [58]/[27] (uncertainties added in quadrature) where green dots/orange diagonal crosses correspond to data and gray squares/lilac crosses correspond to data plus extrapolation to unmeasured energy regions. 
and for $\gamma_{0 n}\left(Q^{2}\right)$ in the region of $0.15<Q^{2}<0.3 \mathrm{GeV}^{2}$. The $\pi \Delta$-loop contribution does not modify the $Q^{2}$ behavior of $\gamma_{0}\left(Q^{2}\right)$ and differs from Ref. [31] by only a small global shift. Note also the relatively large effect of $g_{C}$, which generates a sign change for virtualities above $\sim 0.2 \mathrm{GeV}^{2}$ [see Fig. 3 (upper panel)].

\section{B. $\delta_{L T}\left(Q^{2}\right)$-longitudinal-transverse polarizability}

The longitudinal-transverse spin polarizability,

$$
\begin{aligned}
\delta_{L T}\left(Q^{2}\right) & =\frac{1}{2 \pi^{2}} \int_{\nu_{0}}^{\infty} \mathrm{d} \nu \sqrt{1+\frac{Q^{2}}{\nu^{2}}} \frac{\sigma_{L T}\left(\nu, Q^{2}\right)}{Q \nu^{2}} \\
& =\frac{16 \alpha M_{N}^{2}}{Q^{6}} \int_{0}^{x_{0}} \mathrm{~d} x x^{2}\left[g_{1}\left(x, Q^{2}\right)+g_{2}\left(x, Q^{2}\right)\right],
\end{aligned}
$$

contains information about the spin structure of the nucleon and is another important input in the determination of the (muonic-)hydrogen hyperfine splitting $[16,56]$. It is also relevant in studies of higher-twist corrections to the structure function $g_{2}\left(x, Q^{2}\right)$, given by the moment $d_{2}\left(Q^{2}\right)$ [52] (see Sec. IIIE). The peculiarity of the response encoded in this polarizability is that it involves a spin flip of the nucleon and a polarization change of the photon (see Appendix B and Fig. 11).

It is expected that the Delta isobar gives only a small contribution to $\delta_{L T}\left(Q^{2}\right)$, which makes this polarizability a potentially clean test case for chiral calculations. Consequently, there are relatively many different theoretical calculations of $\delta_{L T}\left(Q^{2}\right)$ coming from different versions of $\chi \mathrm{PT}$ with baryons (HB, IR, and covariant). Reference [51] found a systematic deviation of the HB result for $\delta_{L T n}\left(Q^{2}\right)$ from the MAID model prediction. This disagreement was identified by the authors of Ref. [71] as a puzzle involving the neutron $\delta_{L T}$ polarizability-the $\delta_{L T}$ puzzle. The IR calculation in Ref. [60] also showed a deviation from the data and predicted a rapid rise of $\delta_{L T}\left(Q^{2}\right)$ with growing $Q^{2}$. The problem is solved by keeping the relativistic structure of the theory, as the $\mathrm{B} \chi \mathrm{PT}+\Delta$ result of Ref. [31] showed.

As expected, already the leading $\pi N$ loops provide a reasonable agreement with the experimental data [cf. Fig. 2 (lower panel)]. Since the $\Delta$-exchange contribution to $\delta_{L T}\left(Q^{2}\right)$ is small, the effect of the $g_{M}$ form factor is negligible in this polarizability, as is that of the $g_{C}$ coupling [cf. Fig. 3 (lower panel)]. In fact, we predict both the $\Delta$-exchange and the $\pi \Delta$-loop contributions to be small and negative. This is in agreement with the MAID model, which predicts a small and negative contribution of the $P_{33}$ wave to $\delta_{L T}\left(Q^{2}\right)$. However, in the calculation of Ref. [31], which is different from the one presented here only in the way the $\Delta(1232)$ is included, the contribution of this resonance to $\delta_{L T p}\left(Q^{2}\right)$ is sizable and positive. The authors of that work attributed this large contribution to diagrams where the photons couple directly to the Delta inside a loop. As mentioned in Sec. III A, the effect of such loop diagrams does not change the $Q^{2}$ behavior of the polarizabilities. On the other hand, it can produce a substantial shift of the $\delta_{L T}\left(Q^{2}\right)$ as a whole. A higher-order calculation should resolve the discrepancy between the two covariant approaches; however, it will partially lose the predictive power since the LECs appearing at higher orders will have to be fitted to experimental data.

The $\pi N$-loop, $\Delta$-exchange, and $\pi \Delta$-loop contributions to the NLO $\mathrm{B} \chi \mathrm{PT}$ prediction of the longitudinal-transverse polarizability are, in units of $10^{-4} \mathrm{fm}^{4}$,

$$
\begin{aligned}
& \delta_{L T p}=1.32(15) \approx 1.50-0.16-0.02, \\
& \delta_{L T n}=2.18(23) \approx 2.35-0.16-0.02,
\end{aligned}
$$

while the slopes are, in units of $10^{-4} \mathrm{fm}^{6}$,

$$
\begin{aligned}
& \left.\frac{\mathrm{d} \delta_{L T p}\left(Q^{2}\right)}{\mathrm{d} Q^{2}}\right|_{Q^{2}=0}=-0.85(8) \approx-0.80-0.04-0.01, \\
& \left.\frac{\mathrm{d} \delta_{L T n}\left(Q^{2}\right)}{\mathrm{d} Q^{2}}\right|_{Q^{2}=0}=-1.24(12) \approx-1.19-0.04-0.01 .
\end{aligned}
$$

\section{C. $I_{A}\left(Q^{2}\right)$-a generalized GDH integral}

The helicity-difference cross section $\sigma_{T T}$ exhibits a faster falloff in $\nu$ than its spin-averaged counterpart $\sigma_{T}$. This is due to a cancellation between the leading (constant) terms of $\sigma_{1 / 2}$ and $\sigma_{3 / 2}$ at large $\nu .^{3}$ The resulting $1 / \nu$ falloff of the helicity-difference cross section allows one to write an unsubtracted dispersion relation for the VVCS amplitude $g_{T T}^{\text {nonpole }}\left(\nu, Q^{2}\right)$ [cf. Eq. (10)]. This is the origin of the GDH sum rule $[5,6]$,

$$
-\frac{\alpha}{2 M_{N}^{2}} \varkappa^{2}=\frac{1}{2 \pi^{2}} \int_{\nu_{0}}^{\infty} \mathrm{d} \nu \frac{\sigma_{T T}(\nu)}{\nu},
$$

which establishes a relation to the anomalous magnetic moment $\varkappa$. It is experimentally verified for the nucleon by MAMI (Mainz) and ELSA (Bonn) [72,73].

There are two extensions of the GDH sum rule to finite $Q^{2}$ : the generalized GDH integrals $I_{A}\left(Q^{2}\right)$ and $I_{1}\left(Q^{2}\right)$. The latter will be discussed in Sec. IIID. The former is defined as ${ }^{4}$

\footnotetext{
${ }^{3}$ Notice that a constant term in $\sigma_{T T}$ at $\nu \rightarrow \infty$ is forbidden by crossing symmetry.

${ }^{4}$ Note that $I_{A}\left(Q^{2}\right)$ is sometimes called $I_{T T}\left(Q^{2}\right)$.
} 


$$
\begin{aligned}
& -\frac{\alpha}{2 M_{N}^{2}} I_{A}\left(Q^{2}\right) \\
& =-\frac{1}{8 \pi^{2}} \int_{\nu_{0}}^{\infty} \mathrm{d} \nu \sqrt{1+\frac{Q^{2}}{\nu^{2}}} \frac{\sigma_{T T}\left(\nu, Q^{2}\right)}{\nu} \\
& =\frac{\alpha}{Q^{2}} \int_{0}^{x_{0}} \mathrm{~d} x\left[\frac{4 M_{N}^{2} x^{2}}{Q^{2}} g_{2}\left(x, Q^{2}\right)-g_{1}\left(x, Q^{2}\right)\right] .
\end{aligned}
$$

Because of its energy weighting, the integral in Eq. (26) converges slower than the one in the generalized forward spin polarizability sum rule (19). Therefore, knowledge of the cross section at higher energies is required and the evaluation of the generalized GDH integral $I_{A}\left(Q^{2}\right)$ is not as simple as the evaluation of $\gamma_{0}\left(Q^{2}\right)$.

The generalized GDH integral $I_{A}\left(Q^{2}\right)$ is directly related to the nonpole amplitude $g_{T T}^{\text {nonpole }}\left(\nu, Q^{2}\right)$, which differs from non-Born amplitude $\bar{g}_{T T}\left(\nu, Q^{2}\right)$ by a term involving the elastic Pauli form factor

$$
g_{T T}^{\text {nonpole }}\left(\nu, Q^{2}\right)=\bar{g}_{T T}\left(\nu, Q^{2}\right)-\frac{2 \pi \alpha \nu}{M_{N}^{2}} F_{2}^{2}\left(Q^{2}\right)
$$

[cf. Eqs. (2) and (8)]. Consequently, $I_{A}\left(Q^{2}\right)$ is not a pure polarizability, but also contains an elastic contribution. The "nonpolarizability" or the Born part of $I_{A}\left(Q^{2}\right)$ is given by

$$
I_{A}^{\text {Born }}\left(Q^{2}\right)=I_{A}\left(Q^{2}\right)-\Delta I_{A}\left(Q^{2}\right)=-\frac{1}{4} F_{2}^{2}\left(Q^{2}\right),
$$

where we refer to the polarizability part as $\Delta I_{A}\left(Q^{2}\right)$. The same is true for the generalized GDH integral $I_{1}\left(Q^{2}\right)$, which is directly related to $S_{1}^{\text {nonpole }}\left(\nu, Q^{2}\right)$ :

$$
I_{1}^{\text {Born }}\left(Q^{2}\right)=I_{1}\left(Q^{2}\right)-\Delta I_{1}\left(Q^{2}\right)=-\frac{1}{4} F_{2}^{2}\left(Q^{2}\right) .
$$

In the following, we will add the Born parts to our LO and NLO B $\chi$ PT predictions for the polarizabilities $\Delta I_{A}\left(Q^{2}\right)$ and $\Delta I_{1}\left(Q^{2}\right)$, employing an empirical parametrization for the elastic Pauli form factor [74]. This allows us to compare to the experimental results for $I_{A}\left(Q^{2}\right)$ and $I_{1}\left(Q^{2}\right)$ (cf. Fig. 4). Note that the blue error bands only describe the uncertainties of our $\mathrm{B} \chi \mathrm{PT}$ predictions of the polarizabilities, while the elastic contributions are considered to be exact, as explained in Sec. IIC. The uncertainties of the polarizability predictions are therefore better reflected in Fig. 5, where we show the contributions of the different orders to the $\mathrm{B} \chi \mathrm{PT}$ predictions of $\Delta I_{A}\left(Q^{2}\right)$ and $\Delta I_{1}\left(Q^{2}\right)$, as well as the total results with error bands.

The E97-110 experiment at Jefferson Lab has recently published their data for $I_{A n}\left(Q^{2}\right)$ in the region of $0.035 \mathrm{GeV}^{2}<Q^{2}<0.24 \mathrm{GeV}^{2}$ [27]. In addition, there are results for $I_{A n}\left(Q^{2}\right)$ from the earlier E94-010 experiment
[21], and for $I_{A p}\left(Q^{2}\right)$ from the E08-027 experiment [57]. The $\mathcal{O}\left(p^{4}\right)$ HB calculation gives a large negative effect [52], which does not describe the data. The $\mathrm{B} \chi \mathrm{PT}+\Delta$ result from Ref. [31], which mainly differs from our work by the absence of the dipole form factor in $g_{M}$, looks similar to this HB result and only describes the data points at lowest $Q^{2}$. Our NLO prediction, however, follows closely the $Q^{2}$ evolution of the data. In Fig. 5 (upper panel), we show the polarizability $\Delta I_{A}\left(Q^{2}\right)$, whose $Q^{2}$ evolution is clearly dominated by the $\Delta$ exchange. Similar to the case of $\gamma_{0 p}\left(Q^{2}\right)$, the inclusion of the dipole in $g_{M}$ and the Coulomb coupling $g_{C}$ is very important in order to describe the experimental data. The LO prediction, on the other hand, slightly overestimates the data [cf. Fig. 4 (upper panel)].

At the real-photon point: $I_{A}(0)=-\frac{\varkappa^{2}}{4}$ and $\Delta I_{A}(0)=0$. Therefore, we give only the slope of the polarizability $\Delta I_{A}\left(Q^{2}\right)$ (showing also the separate contributions from $\pi N$ loops, $\Delta$ exchange, and $\pi \Delta$ loops) in units of $\mathrm{GeV}^{-2}$ :

$$
\left.\frac{\mathrm{d} \Delta I_{A p}\left(Q^{2}\right)}{\mathrm{d} Q^{2}}\right|_{Q^{2}=0}=-8.58(3.43) \approx 2.38-11.21+0.25,
$$

$$
\left.\frac{\mathrm{d} \Delta I_{A n}\left(Q^{2}\right)}{\mathrm{d} Q^{2}}\right|_{Q^{2}=0}=-9.55(3.43) \approx 1.41-11.21+0.25 .
$$

Including the empirical Pauli form factor [74], we find, in units of $\mathrm{GeV}^{-2}$,

$$
\left.\frac{\mathrm{d} I_{A p}\left(Q^{2}\right)}{\mathrm{d} Q^{2}}\right|_{Q^{2}=0}=-3.18,\left.\frac{\mathrm{d} I_{A n}\left(Q^{2}\right)}{\mathrm{d} Q^{2}}\right|_{Q^{2}=0}=-3.00 .
$$

\section{D. $\Gamma_{1}\left(Q^{2}\right)$ and $I_{1}\left(Q^{2}\right)$-the first moment of the structure function $g_{1}\left(x, Q^{2}\right)$}

The second variant for a generalization of the GDH sum rule to finite $Q^{2}$ is defined as

$$
\begin{aligned}
- & \frac{\alpha}{2 M_{N}^{2}} I_{1}\left(Q^{2}\right) \\
= & -\frac{1}{8 \pi^{2}} \int_{\nu_{0}}^{\infty} \mathrm{d} \nu \frac{1}{\sqrt{\nu^{2}+Q^{2}}}\left[\sigma_{T T}\left(\nu, Q^{2}\right)+\frac{Q}{\nu} \sigma_{L T}\left(\nu, Q^{2}\right)\right] \\
= & -\frac{\alpha}{Q^{2}} \int_{0}^{x_{0}} \mathrm{~d} x g_{1}\left(x, Q^{2}\right)
\end{aligned}
$$

where $I_{1}(0)=-\frac{\varkappa^{2}}{4}$. This generalized GDH integral directly stems from the amplitude $S_{1}^{\text {nonpole }}\left(\nu, Q^{2}\right)$ with the LEX from Eq. (12). It is given by the first moment of the 

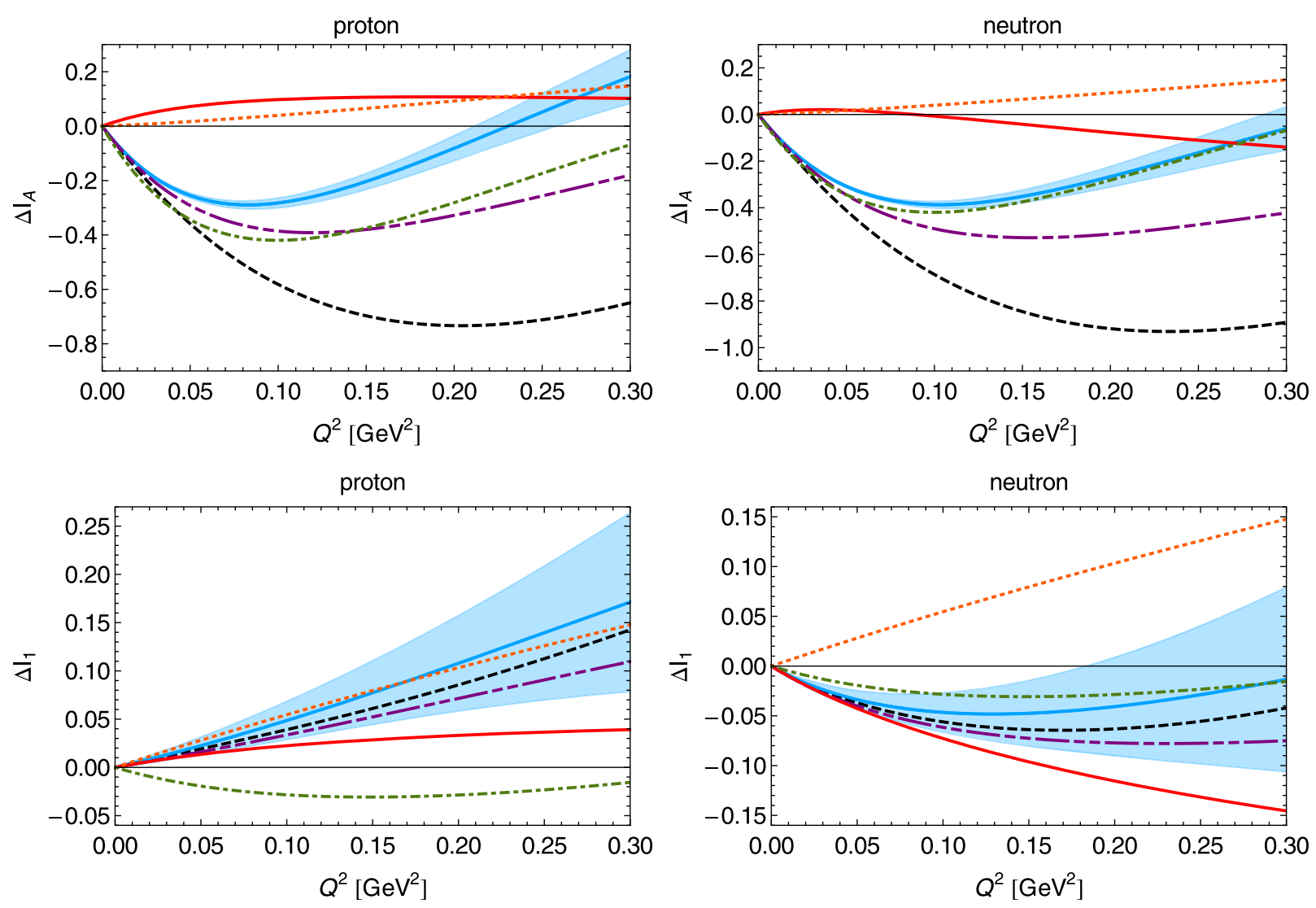

FIG. 5. Contributions of the different orders to the chiral predictions of $\Delta I_{A}\left(Q^{2}\right)$ (upper panel) and $\Delta I_{1}\left(Q^{2}\right)$ (lower panel) for the proton (left) and neutron (right). Red solid line: $\pi N$-loop contribution; green dot-dashed line: $\Delta$-exchange contribution; orange dotted line: $\pi \Delta$-loop contribution; blue solid line and blue band: total result; purple dot-dot-dashed line: total result without $g_{C}$ contribution; and black short-dashed line: total result without $g_{M}$ dipole.

structure function $g_{1}\left(x, Q^{2}\right), \Gamma_{1}\left(Q^{2}\right)=\int_{0}^{x_{0}} \mathrm{~d} x g_{1}\left(x, Q^{2}\right)$, as follows: $I_{1}\left(Q^{2}\right)=\frac{2 M_{N}^{2}}{Q^{2}} \Gamma_{1}\left(Q^{2}\right)$. The isovector combination

$$
\Gamma_{1(p-n)}\left(Q^{2}\right)=\int_{0}^{x_{0}} \mathrm{~d} x\left[g_{1 p}\left(x, Q^{2}\right)-g_{1 n}\left(x, Q^{2}\right)\right]
$$

is related to the axial coupling of the nucleon through the Bjorken sum rule [75,76]:

$$
\lim _{Q^{2} \rightarrow \infty} \Gamma_{1(p-n)}\left(Q^{2}\right)=\frac{g_{A}}{6} .
$$

As explained in Eq. (28), the moment $I_{1}\left(Q^{2}\right)$ splits into a polarizability part $\Delta I_{1}\left(Q^{2}\right)$ and a Born part $I_{1}^{\text {Born }}\left(Q^{2}\right)$. Figure 4 (lower panel) shows the $Q^{2}$ dependence of $I_{1}\left(Q^{2}\right)$ which, in contrast to $I_{A}\left(Q^{2}\right)$ shown in Fig. 4 (upper panel), is clearly dominated by its Born part and the elastic Pauli form factor. The $\pi N$-loop, $\Delta$-exchange, and $\pi \Delta$-loop contributions to the polarizability $\Delta I_{1}\left(Q^{2}\right)$ are shown in Fig. 5 (lower panel). Comparing to Fig. 5 (upper panel), one sees that $\Delta I_{1}\left(Q^{2}\right)$ is less sensitive to $g_{C}$ and the dipole form factor in $g_{M}$ than $\Delta I_{A}\left(Q^{2}\right)$.
For the proton, our NLO $\mathrm{B} \chi \mathrm{PT}$ prediction gives a very good description of the experimental data $[18,57]$ and is in reasonable agreement with the MAID prediction [69]. For the neutron, one observes good agreement with the empirical evaluations including extrapolations to unmeasured energy regions starting from $Q^{2}>0.1 \mathrm{GeV}^{2}$ [27,58]. In the region of $Q^{2}<0.05 \mathrm{GeV}^{2}$, one observes an interesting tension between the recent E97-110 experiment [27] and the data from CLAS [58]. While the newest measurement finds $I_{1 n}\left(0.035 \mathrm{GeV}^{2}\right)<\varkappa_{n}^{2} / 4$, thus suggesting a negative slope at low $Q$, the older measurement found a rather large value for $I_{1 n}\left(0.0496 \mathrm{GeV}^{2}\right)$. A similar but milder behavior is seen in the E97-110 [27] and E94-010 [21] data for $I_{A n}$. The MAID predictions do not agree with the CODATA recommended values for the anomalous magnetic moments of the proton and neutron [70], which in our work are imposed by using empirical parametrizations for the elastic Pauli form factors [74]. The slope of the HB result from Ref. [52] is too large and therefore only reproduces the data at very low $Q^{2}$.

Figure 6 shows the moment $\Gamma_{1}\left(Q^{2}\right)$ for the proton and neutron, while Fig. 7 shows the isovector combination $\Gamma_{1, p-n}\left(Q^{2}\right)$. The LO and NLO $\mathrm{B} \chi \mathrm{PT}$ predictions are 

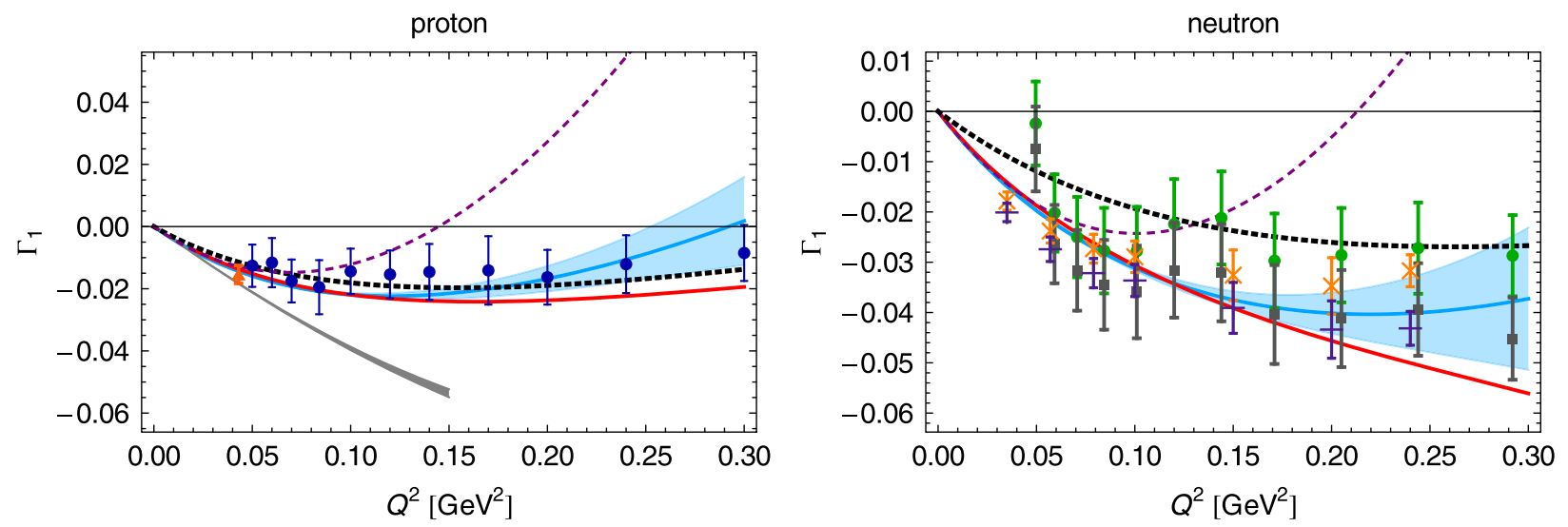

FIG. 6. First moment of the structure function $g_{1}\left(x, Q^{2}\right)$ for the proton (left) and neutron (right) as a function of $Q^{2}$. The legend is the same as in Fig. 4.

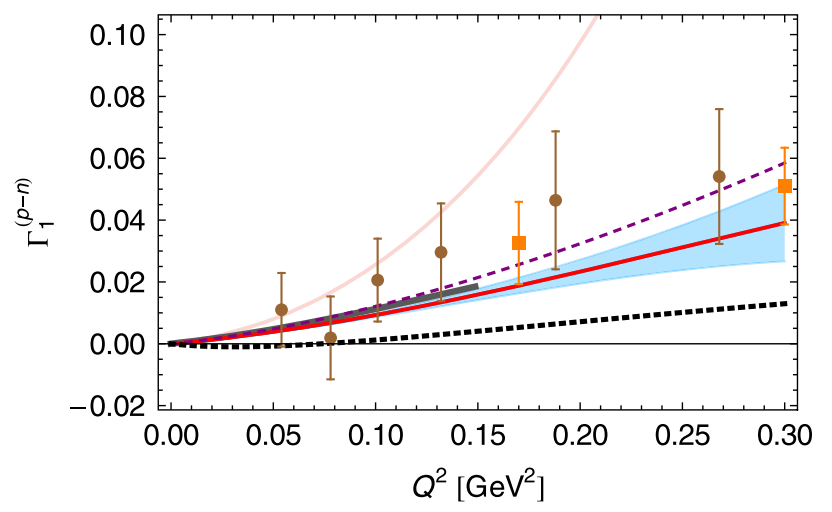

FIG. 7. Isovector combination of $\Gamma_{1}\left(Q^{2}\right)$ as a function of $Q^{2}$. The legend is the same as in Fig. 6 . The pink curve is the IR result from Refs. [60,77]. The experimental points are from Ref. [78] (brown dots) and Ref. [23] (orange squares).

identical, because our calculation produces the same Delta contributions for the proton and the neutron. For the isovector combination, the MAID model only agrees with the data at very low $Q^{2}<0.10 \mathrm{GeV}^{2}$. The same is true for the IR result [60,77], while all other chiral results describe the data: NLO $\mathrm{B} \chi \mathrm{PT}$ (this work), $\mathrm{B} \chi \mathrm{PT}+\Delta[31]$, and $\mathrm{HB} \chi \mathrm{PT}$ [52].

At the real-photon point: $I_{1}(0)=-\frac{\varkappa^{2}}{4}$ and $\Delta I_{1}(0)=0$. Therefore, we give only the slope of the polarizability $\Delta I_{1}\left(Q^{2}\right)$ (showing also the separate contributions from $\pi N$ loops, $\Delta$ exchange, and $\pi \Delta$ loops) in units of $\mathrm{GeV}^{-2}$ :

$\left.\frac{\mathrm{d} \Delta I_{1 p}\left(Q^{2}\right)}{\mathrm{d} Q^{2}}\right|_{Q^{2}=0}=0.39(4) \approx 0.34-0.53+0.58$,

$\left.\frac{\mathrm{d} \Delta I_{1 n}\left(Q^{2}\right)}{\mathrm{d} Q^{2}}\right|_{Q^{2}=0}=-1.01(10) \approx-1.07-0.53+0.58$.
Including the empirical Pauli form factor [74], we find, in units of $\mathrm{GeV}^{-2}$,

$$
\left.\frac{\mathrm{d} I_{1 p}\left(Q^{2}\right)}{\mathrm{d} Q^{2}}\right|_{Q^{2}=0}=5.80,\left.\quad \frac{\mathrm{d} I_{1 n}\left(Q^{2}\right)}{\mathrm{d} Q^{2}}\right|_{Q^{2}=0}=5.53 .
$$

\section{E. $\bar{d}_{2}\left(Q^{\mathbf{2}}\right)$-a measure of color polarizability}

Another interesting moment to consider is $d_{2}\left(Q^{2}\right)$, which is related to the twist-3 part of the spin structure function $g_{2}\left(x, Q^{2}\right)[79,80]$ :

$$
d_{2}\left(Q^{2}\right) \equiv 3 \int_{0}^{1} \mathrm{~d} x x^{2}\left[g_{2}\left(x, Q^{2}\right)-g_{2}^{\mathrm{WW}}\left(x, Q^{2}\right)\right],
$$

where $g_{2}^{\mathrm{WW}}\left(x, Q^{2}\right)$ is the twist-2 part of $g_{2}\left(x, Q^{2}\right)$. Using the Wandzura-Wilczek relation [81], one can relate $d_{2}\left(Q^{2}\right)$ to the moments of the spin structure functions $g_{1}\left(x, Q^{2}\right)$ and $g_{2}\left(x, Q^{2}\right)$ :

$$
d_{2}\left(Q^{2}\right)=\int_{0}^{1} \mathrm{~d} x x^{2}\left[3 g_{2}\left(x, Q^{2}\right)+2 g_{1}\left(x, Q^{2}\right)\right] .
$$

This relation, however, only holds for asymptotically large $Q^{2}$. It is also in the high- $Q^{2}$ region, where $d_{2}\left(Q^{2}\right)$ is a measure of color polarizability [82,83], through its relation to the gluon field strength tensor [80]. We refer to Ref. [84] for a recent review on the spin structure of the nucleon, including a discussion of sum rules for deep inelastic scattering and color polarizabilities.

What we consider in the following is the inelastic part of $d_{2}\left(Q^{2}\right)$, defined as the moment of $g_{1}\left(x, Q^{2}\right)$ and $g_{2}\left(x, Q^{2}\right)$ spin structure functions [cf. Eq. (38)]:

$$
\bar{d}_{2}\left(Q^{2}\right)=\int_{0}^{x_{0}} \mathrm{~d} x x^{2}\left[3 g_{2}\left(x, Q^{2}\right)+2 g_{1}\left(x, Q^{2}\right)\right] .
$$


This moment provides another testing ground for our $\mathrm{B} \chi \mathrm{PT}$ predictions through comparison with experiments on the neutron [22]. Going toward the low- $Q^{2}$ region, the interpretation of $\bar{d}_{2}\left(Q^{2}\right)$ in terms of color polarizabilities will fade out. The above definition, however, implies it is related to other VVCS polarizabilities:

$\bar{d}_{2}\left(Q^{2}\right)=\frac{Q^{4}}{8 M_{N}^{4}}\left[\frac{M_{N}^{2} Q^{2}}{\alpha} \delta_{L T}\left(Q^{2}\right)+I_{1}\left(Q^{2}\right)-I_{A}\left(Q^{2}\right)\right]$.

Note that $\bar{d}_{2}\left(Q^{2}\right)$ and its first two derivatives with respect to $Q^{2}$ vanish at $Q^{2}=0$. The considerations in Eqs. (28) and (29) have no effect on $\bar{d}_{2}\left(Q^{2}\right)$, since the Born contributions from $I_{A}\left(Q^{2}\right)$ and $I_{1}\left(Q^{2}\right)$ cancel out. Therefore, $\bar{d}_{2}\left(Q^{2}\right)$ is a pure polarizability.

In Fig. 8 (upper panel), we show our NLO B $\chi \mathrm{PT}$ prediction and other results for $\bar{d}_{2}\left(Q^{2}\right)$. While MAID [69] and $\mathrm{B} \chi \mathrm{PT}$ describe the experimental data for the neutron [22] very well, the HB limit [51,52] is showing a fast growth with $Q^{2}$. This illustrates the importance of keeping the
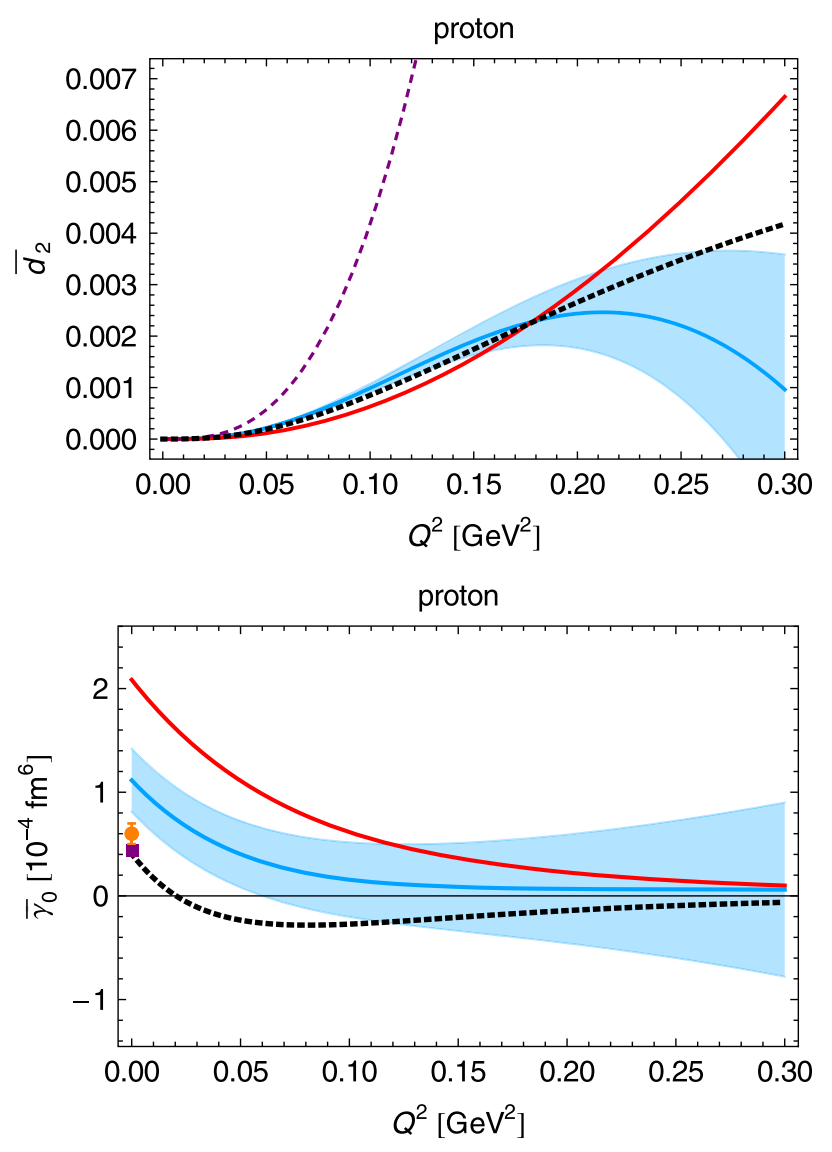

relativistic result. Note also that, even though the $\pi N$-loop contribution is dominant, both $g_{C}$ and the form factor in $g_{M}$ are essential to obtain a curvature that reproduces the data [cf. Fig. 9 (upper panel)]. For the proton there are, to our knowledge, no experimental results to compare with. However, the agreement between the NLO B $\chi$ PT prediction and the MAID prediction at low energies is reasonable.

\section{F. $\bar{\gamma}_{0}\left(Q^{2}\right)$-fifth-order generalized forward spin polarizability}

It is interesting to compare the generalized fifth-order forward spin polarizability sum rule,

$$
\begin{aligned}
\bar{\gamma}_{0}\left(Q^{2}\right) & =\frac{1}{2 \pi^{2}} \int_{\nu_{0}}^{\infty} \mathrm{d} \nu \sqrt{1+\frac{Q^{2}}{\nu^{2}}} \frac{\sigma_{T T}\left(\nu, Q^{2}\right)}{\nu^{5}} \\
& =\frac{64 \alpha M_{N}^{4}}{Q^{10}} \int_{0}^{x_{0}} \mathrm{~d} x x^{4}\left[g_{1}\left(x, Q^{2}\right)-\frac{4 M_{N}^{2} x^{2}}{Q^{2}} g_{2}\left(x, Q^{2}\right)\right],
\end{aligned}
$$
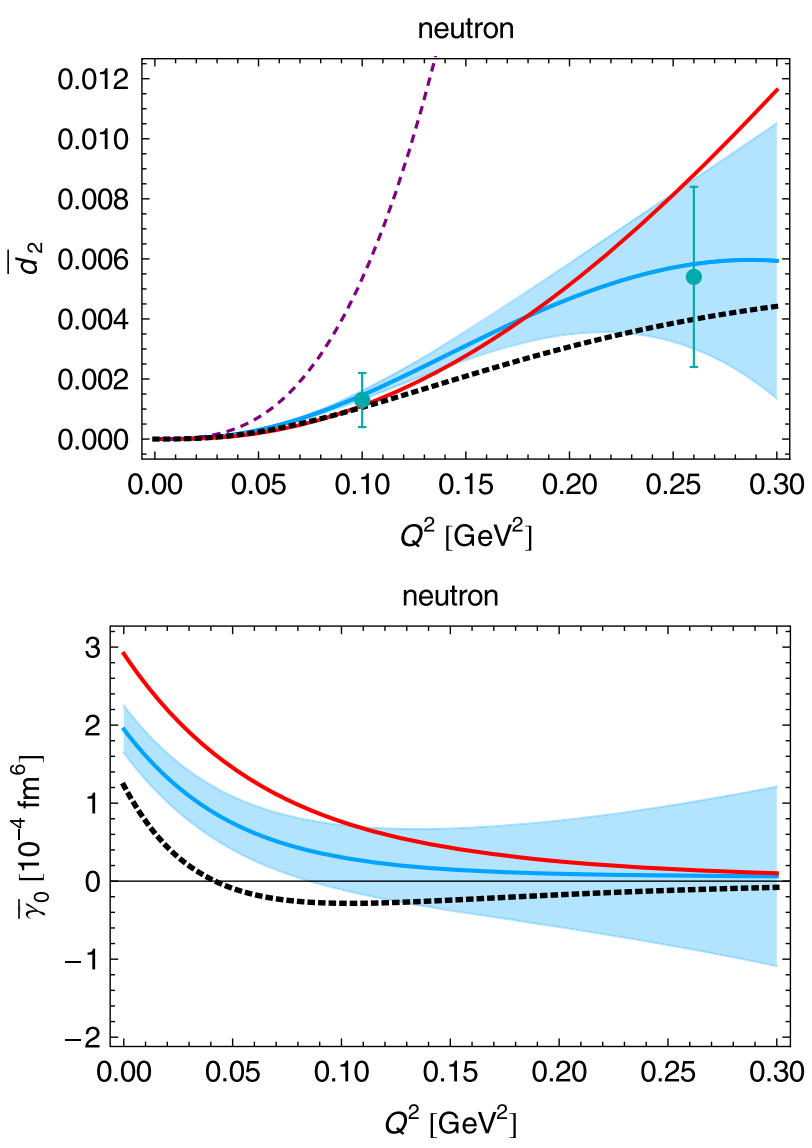

FIG. 8. Upper panel: The inelastic moment $\bar{d}_{2}\left(Q^{2}\right)$ for the proton (left) and neutron (right) as a function of $Q^{2}$. The result of this work, the NLO B $\chi$ PT prediction, is shown by the blue solid line and the blue band. The red line represents the LO B $\chi$ PT result. The purple short-dashed line is the $\mathcal{O}\left(p^{4}\right) \mathrm{HB}$ result from Refs. [51,52]. The black dotted line is the MAID model prediction [69]. The experimental points for the neutron (cyan dots) are from Ref. [22]. Lower panel: Fifth-order generalized forward spin polarizability $\bar{\gamma}_{0}\left(Q^{2}\right)$, for the proton (left) and neutron (right) as a function of $Q^{2}$. The experimental points for the proton are from Ref. [64] (purple square) and Ref. [85] (orange dot). 

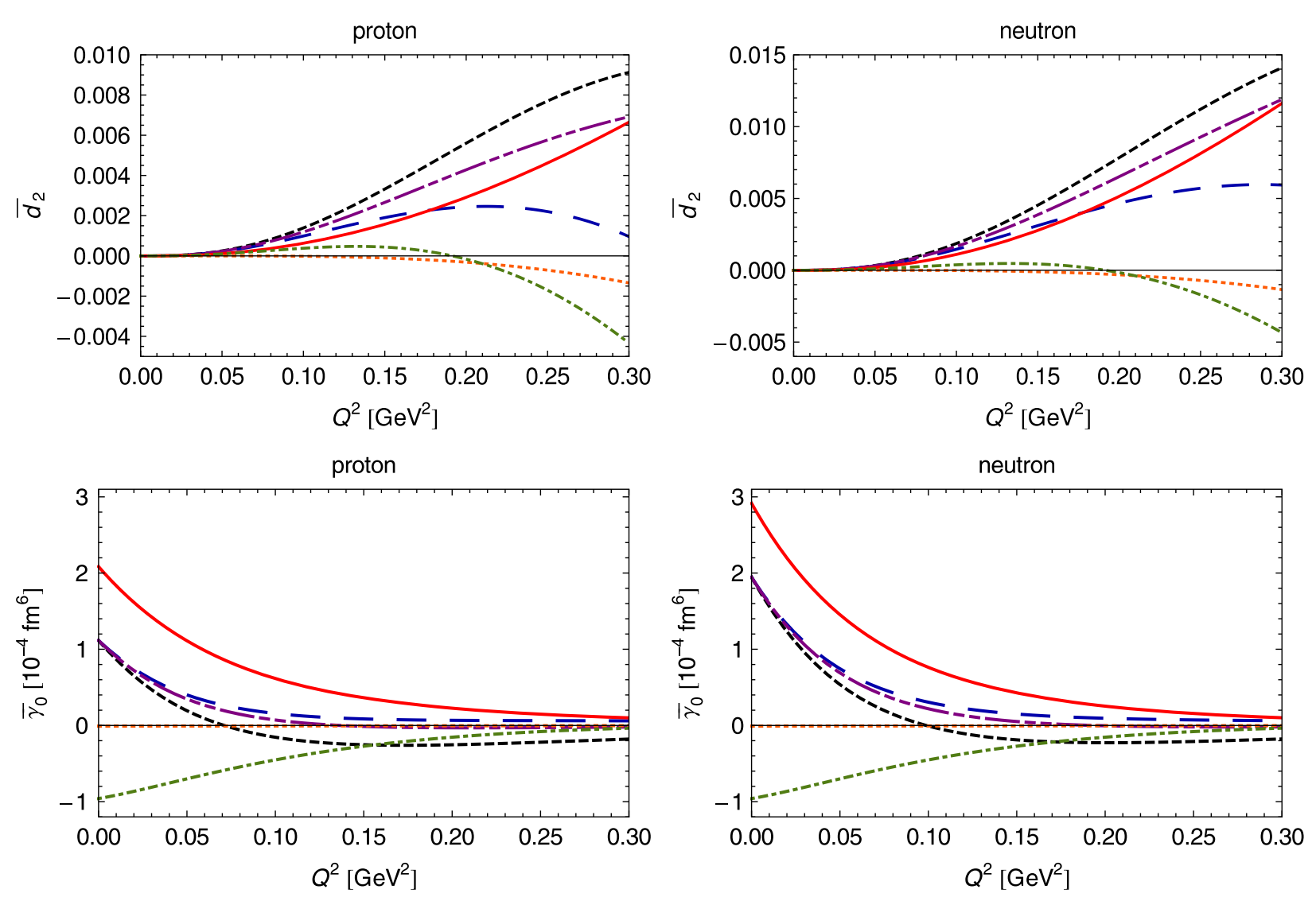

FIG. 9. Contributions of the different orders to the chiral predictions of $\bar{d}_{2}\left(Q^{2}\right)$ (upper panel) and $\bar{\gamma}_{0}\left(Q^{2}\right)$ (lower panel) for the proton (left) and neutron (right). Red solid line: $\pi N$-loop contribution; green dot-dashed line: $\Delta$-exchange contribution; orange dotted line: $\pi \Delta$-loop contribution; blue long-dashed line: total result; purple dot-dot-dashed line: total result without $g_{C}$ contribution; and black short-dashed line: total result without $g_{M}$ dipole.

to the sum rule integrals for $I_{A}\left(Q^{2}\right)$ and $\gamma_{0}\left(Q^{2}\right)$, since they differ merely by their energy weighting of $\sigma_{T T}\left(\nu, Q^{2}\right)$ and a constant prefactor [cf. Eqs. (19), (26), and (41)]. From $I_{A}\left(Q^{2}\right)$ to $\gamma_{0}\left(Q^{2}\right)$ to $\bar{\gamma}_{0}\left(Q^{2}\right)$, the energy suppression is increasing by a factor of $\nu^{-2}$, respectively. Therefore, the description of $\bar{\gamma}_{0}\left(Q^{2}\right)$ should be easiest in a low-energy effective-field theory such as $\chi \mathrm{PT}$, whereas $\gamma_{0}\left(Q^{2}\right)$ and $I_{A}\left(Q^{2}\right)$ receive larger contributions from higher energies.

In Fig. 8 (lower panel), we show our LO and NLO B $\chi$ PT predictions for $\bar{\gamma}_{0}\left(Q^{2}\right)$. One can see that the $\pi N$-loop contribution is positive (in accordance to what we see for the cross section $\sigma_{T T}$; see Fig. 10). The Delta shifts it substantially, especially in the low $Q^{2}$ region, bringing it into a better agreement with data. In general, the $\mathrm{B} \chi \mathrm{PT}$ curves start above the empirical data points at the realphoton point, and then decrease asymptotically to zero above $Q^{2}>0.1 \mathrm{GeV}^{2}$. On the other hand, the MAID prediction reproduces the empirical data at the real-photon point, then decreases to negative values until about $Q^{2}>0.06 \mathrm{GeV}^{2}$, from where it also starts to asymptotically approach zero. Consequently, our NLO B $\chi$ PT prediction of $\bar{\gamma}_{0}\left(Q^{2}\right)$ is consistently above the MAID prediction. This is very different from what we saw for $I_{A}\left(Q^{2}\right)$ in Fig. 4 (upper panel), where the MAID prediction at the real-photon point is above the experimental value. While the agreement of our predictions with the empirical data is in general quite good for all moments of $\sigma_{T T}\left(\nu, Q^{2}\right)$, one should point out that both for $\gamma_{0 n}\left(Q^{2}\right)$ and $\bar{\gamma}_{0 p}\left(Q^{2}\right)$ we overestimate the data at low $Q^{2}$. For $I_{A}\left(Q^{2}\right)$ such an observation cannot be made because $\Delta I_{A}(0)=0$, and thus, $I_{A}(0)$ is given by the empirical Pauli form factor only. From $I_{A}\left(Q^{2}\right), \gamma_{0}\left(Q^{2}\right)$, and $\bar{\gamma}_{0}\left(Q^{2}\right)$, the latter has the smallest, however, non-negligible dependence on $g_{C}$ and the dipole in $g_{M}$ [cf. Fig. 9 (lower panel)].

The $\pi N$-loop, $\Delta$-exchange, and $\pi \Delta$-loop contributions to the NLO $\mathrm{B} \chi \mathrm{PT}$ prediction of the fifth-order forward spin polarizability amount to, in units of $10^{-4} \mathrm{fm}^{6}$,

$$
\begin{aligned}
& \bar{\gamma}_{0 p}=1.12(30) \approx 2.08-0.96-0.01, \\
& \bar{\gamma}_{0 n}=1.95(30) \approx 2.92-0.96-0.01,
\end{aligned}
$$

while the slope is composed as follows, in units of $10^{-4} \mathrm{fm}^{8}$ : 
TABLE II. The NLO B $\chi$ PT predictions for the forward VVCS polarizabilities and their slopes at $Q^{2}=0$. The contributions of the $\pi N$ loops, the $\Delta$ exchange, and the $\pi \Delta$ loops are shown, together with the combined total result. Note that $I_{A}(0)=$ $I_{1}(0)=\bar{d}_{2}(0)=0$ and $\left(\bar{d}_{2}\right)^{\prime}=0$.

\begin{tabular}{|c|c|c|c|c|c|}
\hline & & $\pi N$ loops & $\Delta$ exchange & $\pi \Delta$ loops & Total \\
\hline $\begin{array}{l}\gamma_{0} \\
\left(10^{-4} \mathrm{fm}^{4}\right)\end{array}$ & $\begin{array}{l}p \\
n\end{array}$ & $\begin{array}{l}2.01 \\
2.98\end{array}$ & -2.84 & -0.10 & $\begin{array}{l}-0.93(92) \\
0.03(92)\end{array}$ \\
\hline $\begin{array}{l}\delta_{L T} \\
\left(10^{-4} \mathrm{fm}^{4}\right)\end{array}$ & $\begin{array}{l}p \\
n\end{array}$ & $\begin{array}{l}1.50 \\
2.35\end{array}$ & -0.16 & -0.02 & $\begin{array}{l}1.32(15) \\
2.18(23)\end{array}$ \\
\hline $\begin{array}{l}\bar{\gamma}_{0} \\
\left(10^{-4} \mathrm{fm}^{6}\right)\end{array}$ & $\begin{array}{l}p \\
n\end{array}$ & $\begin{array}{l}2.08 \\
2.92\end{array}$ & -0.96 & -0.01 & $\begin{array}{l}1.12(30) \\
1.95(30)\end{array}$ \\
\hline $\begin{array}{l}\left(\gamma_{0}\right)^{\prime} \\
\left(10^{-4} \mathrm{fm}^{6}\right)\end{array}$ & $\begin{array}{l}p \\
n\end{array}$ & $\begin{array}{l}-0.33 \\
-0.73\end{array}$ & 0.11 & 0.01 & $\begin{array}{l}-0.22(4) \\
-0.61(7)\end{array}$ \\
\hline $\begin{array}{l}\left(\delta_{L T}\right)^{\prime} \\
\left(10^{-4} \mathrm{fm}^{6}\right)\end{array}$ & $\begin{array}{l}p \\
n\end{array}$ & $\begin{array}{l}-0.80 \\
-1.19\end{array}$ & -0.04 & -0.01 & $\begin{array}{l}-0.85(8) \\
-1.24(12)\end{array}$ \\
\hline $\begin{array}{l}\left(\bar{\gamma}_{0}\right)^{\prime} \\
\left(10^{-4} \mathrm{fm}^{8}\right)\end{array}$ & $\begin{array}{l}p \\
n\end{array}$ & $\begin{array}{l}-1.00 \\
-1.58\end{array}$ & 0.16 & 0.00 & $\begin{array}{l}-0.84(10) \\
-1.42(15)\end{array}$ \\
\hline $\begin{array}{l}\left(\Delta I_{A}\right)^{\prime} \\
\left(\mathrm{GeV}^{-2}\right)\end{array}$ & $\begin{array}{l}p \\
n\end{array}$ & $\begin{array}{l}2.38 \\
1.41\end{array}$ & -11.21 & 0.25 & $\begin{array}{l}-8.58(3.43) \\
-9.55(3.43)\end{array}$ \\
\hline $\begin{array}{l}\left(\Delta I_{1}\right)^{\prime} \\
\left(\mathrm{GeV}^{-2}\right)\end{array}$ & $\begin{array}{l}p \\
n\end{array}$ & $\begin{array}{c}0.34 \\
-1.07\end{array}$ & -0.53 & 0.58 & $\begin{array}{l}0.39(4) \\
-1.01(10)\end{array}$ \\
\hline
\end{tabular}

$$
\left.\frac{\mathrm{d} \bar{\gamma}_{0 p}\left(Q^{2}\right)}{\mathrm{d} Q^{2}}\right|_{Q^{2}=0}=-0.84(10) \approx-1.00+0.16+0.00
$$

$$
\left.\frac{\mathrm{d} \bar{\gamma}_{0 n}\left(Q^{2}\right)}{\mathrm{d} Q^{2}}\right|_{Q^{2}=0}=-1.42(15) \approx-1.58+0.16+0.00
$$

Note that the HB prediction of $\bar{\gamma}_{0 p}\left[4.23\right.$ at $\mathcal{O}\left(p^{3}\right)$ and 3.65 at $\left.\mathcal{O}\left(\epsilon^{3}\right)[85,86]\right]$ is almost an order of magnitude larger than the empirical value, and therefore not shown in Fig. 8.

\section{G. Summary}

Our results are summarized in Table II, where we give the contributions of the different orders to the chiral predictions of the polarizabilities and their slopes at the real-photon point. A quantitative comparison of our predictions for the spin polarizabilities to the work of Bernard et al. [31] and different empirical evaluations is shown in Table III. We can see that the inclusion of the Delta turns out to be very important for all moments of the helicitydifference cross section. To describe the $Q^{2}$ behavior of the polarizabilities, the magnetic coupling of the $N \rightarrow \Delta$ transition should be modified by a dipole form factor, as has been observed previously in the description of electroproduction data [33]. This dipole form factor effectively takes account of vector-meson exchanges. The Coulombquadrupole $N \rightarrow \Delta$ transition, despite its subleading order, is important in the description of some moments of spin structure functions. This is contrary to what we saw for the moments of unpolarized structure functions [30], where the Coulomb coupling had a negligible effect. The $\pi \Delta$ loops are mainly relevant for the generalized GDH integrals.

\section{CONCLUSIONS}

We have presented a complete NLO calculation of the polarized non-Born VVCS amplitudes in covariant $\mathrm{B} \chi \mathrm{PT}$, with pion, nucleon, and $\Delta(1232)$ fields. The dispersion relations between the VVCS amplitudes and the tree-level photoabsorption cross sections served as a cross-check of these calculations.

The obtained moments of the proton and neutron spin structure functions, related to generalized polarizabilities and GDH-type integrals, agree well with the available experimental data. The description of their $Q^{2}$ evolution is improved compared to the previous $\chi \mathrm{PT}$ predictions. In particular, the NLO $\mathrm{B} \chi \mathrm{PT}$ predictions obtained here give a better description of the empirical data (e.g., from the Jefferson Laboratory "Spin Physics Program") than the HB [51,52] and IR [60] calculations.

TABLE III. Our NLO B $\chi \mathrm{PT}$ predictions for the spin polarizabilities at $Q^{2}=0$, compared with the $\mathrm{B} \chi \mathrm{PT}+\Delta$ predictions from Bernard et al. [31], and the available empirical information. Where the reference is not given, the

\begin{tabular}{|c|c|c|c|c|c|c|}
\hline & \multicolumn{3}{|c|}{ Proton } & \multicolumn{3}{|c|}{ Neutron } \\
\hline & This work & $\mathrm{B} \chi \mathrm{PT}+\Delta$ & Empirical & This work & $\mathrm{B} \chi \mathrm{PT}+\Delta$ & Empirical \\
\hline $\begin{array}{l}\gamma_{0} \\
\left(10^{-4} \mathrm{fm}^{4}\right)\end{array}$ & $-0.93(92)$ & $-1.74(40)$ & $\begin{array}{l}-1.00(8)(12)[19] \\
-0.90(8)(11)[85] \\
-0.929(105)[64]\end{array}$ & $0.03(92)$ & $-0.77(40)$ & $\begin{array}{c}-0.005 \\
{[\mathrm{MAID}]}\end{array}$ \\
\hline $\begin{array}{l}\delta_{L T} \\
\left(10^{-4} \mathrm{fm}^{4}\right)\end{array}$ & $1.32(15)$ & $2.40(1)$ & $\begin{array}{c}1.34 \\
\text { [MAID] }\end{array}$ & $2.18(23)$ & $2.38(3)$ & $\begin{array}{c}2.03 \\
{[\mathrm{MAID}]}\end{array}$ \\
\hline $\begin{array}{l}\bar{\gamma}_{0} \\
\left(10^{-4} \mathrm{fm}^{6}\right)\end{array}$ & $1.12(30)$ & & $\begin{array}{l}0.60(7)(7)[85] \\
0.484(82)[64]\end{array}$ & $1.95(30)$ & & $\begin{array}{c}1.23 \\
\text { [MAID] }\end{array}$ \\
\hline
\end{tabular}
empirical number is provided by the MAID analysis [61,69] with unspecified uncertainty. 
The demonstrated predictive power of the $\chi \mathrm{PT}$ framework amplitudes makes it well suited for extending the $\chi \mathrm{PT}$ evaluation of the TPE effect in the hyperfine structure of (muonic-)hydrogen [15-17] to next-to-leading order.

\section{ACKNOWLEDGMENTS}

We thank Lothar Tiator and Marc Vanderhaeghen for helpful discussions. This work is supported by the Deutsche Forschungsgemeinschaft (DFG) through the Collaborative Research Center [The Low-Energy Frontier of the Standard Model (SFB 1044)]. J. M. A. acknowledges support from the Community of Madrid through the "Programa de atracción de talento investigador 2017 (Modalidad 1)," and the Spanish MECD Grant No. FPA2016-77313-P. F. H. gratefully acknowledges financial support from the Swiss National Science Foundation.

\section{APPENDIX A: TENSOR DECOMPOSITIONS OF THE VVCS AMPLITUDES}

In this Appendix, we review the decomposition of the forward VVCS process into tensor structures and scalar amplitudes. In particular, we consider the connection between the covariant and the semirelativistic decomposition in the lab frame that is defined in terms of the conventional transverse, longitudinal, transversetransverse, and transverse-longitudinal amplitudes.

As explained in Sec. II A, the process of forward VVCS off the nucleon can be described in terms of four explicitly covariant amplitudes $S_{1,2}$ and $T_{1,2}$ [3]:

$$
\begin{aligned}
T\left(\nu, Q^{2}\right)= & \left\{\left(-g^{\mu \nu}+\frac{q^{\mu} q^{\nu}}{q^{2}}\right) T_{1}\left(\nu, Q^{2}\right)+\frac{1}{M_{N}^{2}}\left(p^{\mu}-\frac{p \cdot q}{q^{2}} q^{\mu}\right)\left(p^{\nu}-\frac{p \cdot q}{q^{2}} q^{\nu}\right) T_{2}\left(\nu, Q^{2}\right)\right. \\
& \left.-\frac{1}{M_{N}} \gamma^{\mu \nu \alpha} q_{\alpha} S_{1}\left(\nu, Q^{2}\right)-\frac{1}{M_{N}^{2}}\left(\gamma^{\mu \nu} q^{2}+q^{\mu} \gamma^{\nu \alpha} q_{\alpha}-q^{\nu} \gamma^{\mu \alpha} q_{\alpha}\right) S_{2}\left(\nu, Q^{2}\right)\right\} \epsilon_{\mu}^{\prime *} \epsilon_{\nu},
\end{aligned}
$$

where $\epsilon_{\mu}\left(\epsilon_{\mu}^{\prime *}\right)$ are the incoming (outgoing) photon polarization vectors, $\nu$ is the photon lab-frame energy, and $Q^{2}$ is the photon virtuality. Alternatively, the decomposition in the laboratory frame (which in the forward case coincides with the Breit frame) is parametrized in terms of the nucleon Pauli matrices $\vec{\sigma}$ and the four scalar functions $f_{L}, f_{T}, g_{T T}$, and $g_{L T}$ :

$$
T\left(\nu, Q^{2}\right)=\varepsilon_{0} \varepsilon_{0}^{*} f_{L}\left(\nu, Q^{2}\right)+\left(\vec{\varepsilon}^{*} \cdot \vec{\varepsilon}\right) f_{T}\left(\nu, Q^{2}\right)+i \vec{\sigma} \cdot\left(\vec{\varepsilon}^{*} \times \vec{\varepsilon}\right) g_{T T}\left(\nu, Q^{2}\right)-i \vec{\sigma} \cdot\left[\left(\varepsilon_{0} \vec{\varepsilon}^{*}-\vec{\varepsilon} \varepsilon_{0}^{* *}\right) \times \hat{q}\right] g_{L T}\left(\nu, Q^{2}\right) .
$$

Here, $\vec{q}$ and $\hat{q}=\vec{q} /|\vec{q}|$ are the photon three-momentum in the lab system and its unit vector. The modified polarization vector components are given by

$$
\varepsilon_{0}=\left[\epsilon_{0}-\frac{\nu}{|\vec{q}|}(\vec{\epsilon} \cdot \hat{q})\right] \frac{|\vec{q}|}{Q}, \quad \vec{\varepsilon}=\vec{\epsilon}-\hat{q}(\vec{\epsilon} \cdot \hat{q})
$$

where $\epsilon=\left(\epsilon_{0}, \vec{\epsilon}\right)$ is the usual incoming photon polarization vector, and $\epsilon^{\prime *}$ the outgoing polarization vector. The LEX of the lab frame amplitudes [Eq. (10)] can serve, in particular, as the definition of the generalized polarizabilities. The lab frame amplitudes are also conveniently used for the definition of the response functions; see the example of the scalar amplitude $g_{L T}\left(\nu, Q^{2}\right)$ and the corresponding response function $\sigma_{L T}\left(\nu, Q^{2}\right)$ below in Appendix B.

\section{APPENDIX B: PHOTOABSORPTION CROSS SECTIONS}

In the forward kinematics, the spin-dependent VVCS amplitudes and the spin polarizabilities can be described in terms of the polarized structure functions $g_{1}\left(x, Q^{2}\right)$ and $g_{2}\left(x, Q^{2}\right)$, or equivalently, the helicity-difference cross section $\sigma_{T T}\left(\nu, Q^{2}\right)$ and the longitudinal-transverse response function $\sigma_{L T}\left(\nu, Q^{2}\right)$, with the help of dispersion relations (5) and the optical theorem (3). In this way, the photoabsorption cross sections, measured in electroproduction processes, form the basis for the most empirical evaluations shown throughout Sec. III. In the following, we present the $\mathrm{B} \chi \mathrm{PT}$ predictions for the tree-level cross sections of $\pi N-$, $\pi \Delta$-, and $\Delta$-production through photoabsorption on the nucleon (cf. Figs. 8, 9, and 10 in Ref. [30]). In Secs. B 1 and $\mathrm{B} 2$, we will discuss the leading $\pi N$-production channel and the $\Delta$-production channel, respectively. We used these cross sections to verify the polarizability predictions obtained otherwise from the calculated non-Born VVCS amplitudes. Because of the bad high-energy behavior of the $\pi \Delta$-production cross sections in $\mathrm{B} \chi \mathrm{PT}$ (cf. Fig. 10), the dispersion relations in Eq. (5) require further subtractions for a reconstruction of the $\pi \Delta$-loop contribution to the spin-dependent VVCS amplitudes. Therefore, not all polarizabilities could be verified, but only those appearing as higher-order terms in the LEX of the VVCS amplitudes, such as $\bar{\gamma}_{0}[16]$. 

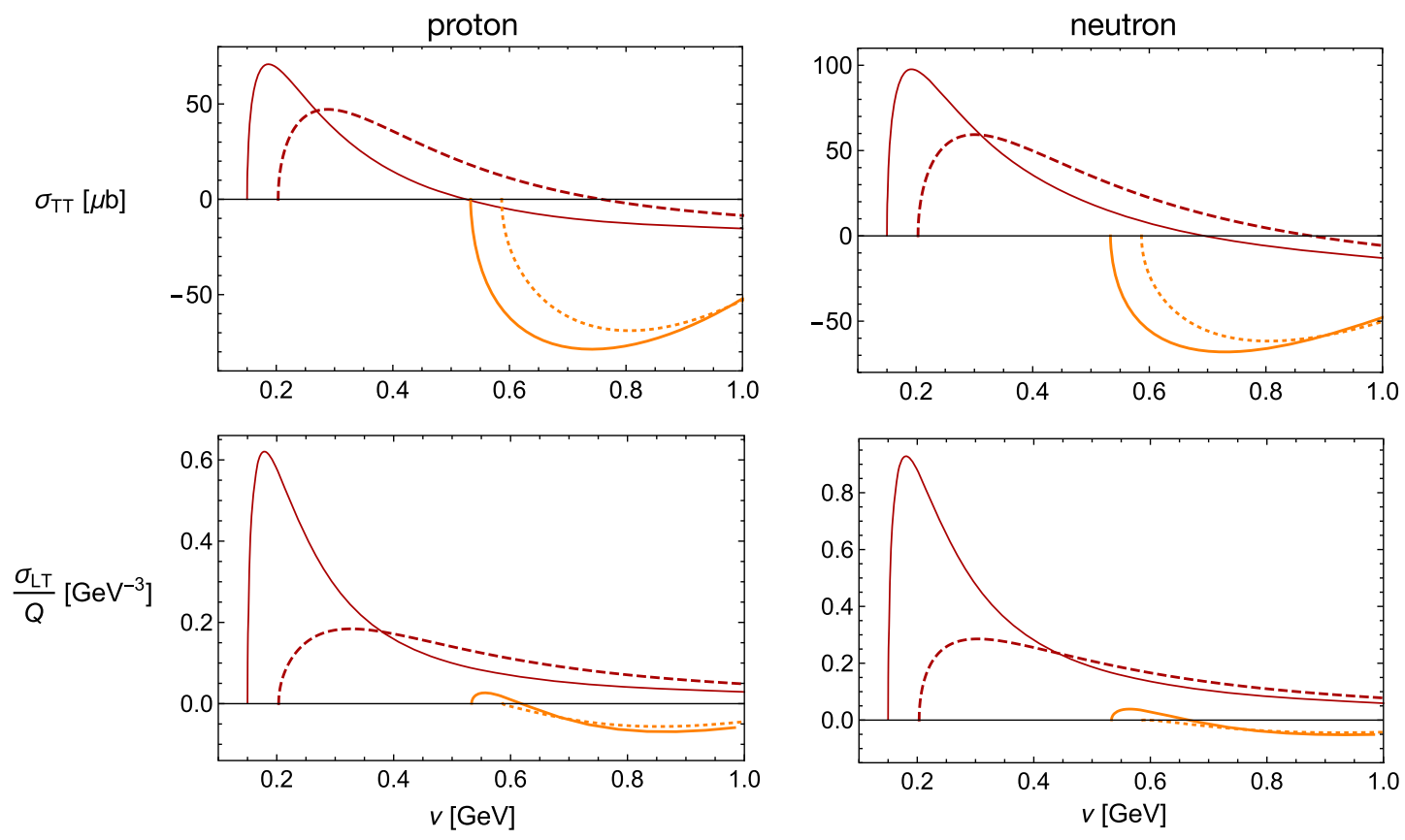

FIG. 10. Photoabsorption cross sections for $\pi N$ (red lines) and $\pi \Delta$ production (orange lines) with $Q^{2}=0$ (solid lines) and $Q^{2}=0.1 \mathrm{GeV}^{2}$ (dashed lines for $\pi N$ and dotted lines for $\pi \Delta$ channels).

\section{1. $\pi N$-production channel}

In order to extract the response function $\sigma_{L T}\left(\nu, Q^{2}\right)$, we have developed a method similar to the one used to calculate $\sigma_{T T}\left(\nu, Q^{2}\right)$ (see, for example, Ref. [87]). For $\sigma_{L T}\left(\nu, Q^{2}\right)$, however, the calculation is more complicated because one has to take into account that the associated Compton process involves a spin flip of the nucleon, as illustrated in Fig. 11. When calculating the cross section, the product of the incoming nucleon spinors has to reflect this flip.

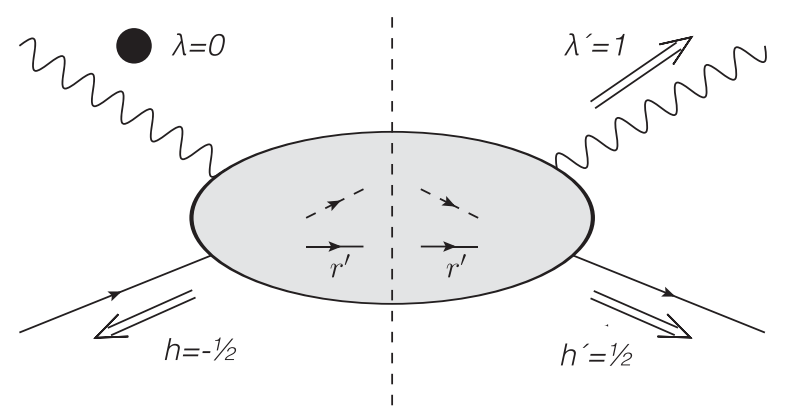

FIG. 11. Relation between the forward Compton process and the photoabsorption process given by the optical theorem. In particular, we show the longitudinal-transverse contribution. The double-line arrows represent the spin of the external particles, while the dot represents the scalar (longitudinal) polarization of the incoming photon. Inside the blob the intermediate states are represented: e.g., nucleons with spins $r^{\prime}$ (which are averaged in the calculation of the cross section) and pions.
The forward VVCS amplitude related to $\sigma_{L T}\left(\nu, Q^{2}\right)$ and $\delta_{L T}\left(Q^{2}\right)$-is $g_{L T}\left(\nu, Q^{2}\right)$. It can be extracted from Eq. (A2) if one takes the modified polarization vector components in Eq. (A3) with $\epsilon=\epsilon_{L}$ and $\epsilon^{\prime *}=\epsilon_{ \pm}^{*}$ as input, where $\epsilon_{L}=\frac{1}{Q}\left(|\vec{q}|, 0,0, q^{0}\right)$ and $\epsilon_{ \pm}=\mp \frac{1}{\sqrt{2}}(0,1, \pm i, 0)$ are the standard longitudinal and transverse polarization vectors, respectively. For $\epsilon_{L}$ and $\epsilon_{ \pm}^{*}$, only the choice of helicities $h^{\prime}= \pm 1 / 2$ and $h=\mp 1 / 2$ gives a nonzero contribution, and one obtains

$$
\begin{aligned}
\chi_{h^{\prime}}^{\dagger} T\left(\nu, Q^{2}\right) \chi_{h} & =\chi_{h^{\prime}}^{\dagger}\left\{-i \vec{\sigma} \cdot\left[\left(\varepsilon_{0} \vec{\varepsilon}^{*}-\vec{\varepsilon} \varepsilon_{0}^{*}\right) \times \hat{q}\right] g_{L T}\left(\nu, Q^{2}\right)\right\} \chi_{h} \\
& =\sqrt{2} g_{L T}\left(\nu, Q^{2}\right),
\end{aligned}
$$

where $\chi_{h}$ and $\chi_{h^{\prime}}^{\dagger}$ are two-component Pauli spinors with opposite helicities, or here, spins.

Let us now consider the related photoabsorption process and, in particular, the tree-level $\gamma^{*} N \rightarrow \pi N$ channel (see diagrams in Fig. 8 of Ref. [30]). We define the $\pi N$-production amplitude as

$$
\mathcal{T}=\bar{u}_{h_{B}}\left(P_{B}\right) \sum_{i} \mathcal{A}_{i}(s, t) \Gamma_{i} u_{h_{A}}\left(P_{A}\right),
$$

with the Dirac structures

$$
\begin{aligned}
& \Gamma_{1}=\gamma_{5}, \\
& \Gamma_{2}=\frac{1}{2}\left[\phi_{A}, \phi\right] \gamma_{5},
\end{aligned}
$$


where $u_{h_{A}}\left(P_{A}\right)$ and $u_{h_{B}}^{\dagger}\left(P_{B}\right)$ are the Dirac spinors and $P_{A}$ and $P_{B}$ are the four-momenta of the incoming and outgoing nucleons, respectively. When calculating the photoabsorption cross section, related to the VVCS amplitude in Eq. (B1), the nucleon spin flip should be implemented by $\bar{u}_{h^{\prime}}\left(P_{A}\right)$ in $\mathcal{T}^{\dagger}$ and $u_{h}\left(P_{A}\right)$ in $\mathcal{T}$, together with the appropriate transverse and longitudinal photon polarization vectors $\epsilon_{ \pm}^{*}$ and $\epsilon_{L}$.

However, if one wants to use the properties of the Dirac matrices, it is more useful to construct an operator to produce this spin flip in the external nucleons of Fig. 11. This is accomplished by introducing the projector $\Gamma_{L T} \equiv \frac{1}{2 \sqrt{2}}\left(\gamma^{1}+i \gamma^{2}\right) \gamma_{5}$, which also takes into account the extra factor $\sqrt{2}$ in Eq. (B1). We checked that with this projector one correctly extracts $\delta_{L T}$ by comparing the HB limit of our result to the HB result of Ref. [51], where the authors calculate this polarizability from the Compton amplitude directly. With all those ingredients, the longitudinal-transverse cross section is calculated in the following way:

$\sigma_{L T}\left(\nu, Q^{2}\right)=\frac{1}{64 \pi^{2} s} \frac{\left|\vec{p}_{f}\right|_{\mathrm{cm}}}{\left|\vec{p}_{i}\right|_{\mathrm{cm}}} \int_{-1}^{1} \mathrm{~d} \cos \theta \sum_{i, j} \mathcal{A}_{i} \mathcal{A}_{j}^{\dagger} \mathcal{X}_{i j}$

with

$$
\mathcal{X}_{i j}=\operatorname{Tr}\left[\left(\not P_{B}+M_{N}\right) \Gamma_{i}\left(\not P_{A}+M_{N}\right) \Gamma_{L T} \gamma^{0} \Gamma_{j}^{\dagger} \gamma^{0}\right],
$$

where $\theta$ is the scattering angle in the center-of-mass $(\mathrm{cm})$ frame and $\left|\vec{p}_{i}\right|_{\mathrm{cm}}\left(\left|\vec{p}_{f}\right|_{\mathrm{cm}}\right)$ is the three-momentum of an incoming (outgoing) particle in the $\mathrm{cm}$ frame. An explicit calculation of the matrix $\mathcal{X}_{i j}$ leads to

$$
\mathcal{X}=M_{N} Q\left(\begin{array}{cc}
0 & 2\left(P_{B}-P_{A}\right) \cdot \epsilon_{L} \\
-\sqrt{2}\left|\vec{q}_{f}\right|_{\mathrm{cm}} \sin \theta & (s-u)
\end{array}\right),
$$

where $\left|\vec{q}_{i}\right|_{\mathrm{cm}}\left(\left|\vec{q}_{f}\right|_{\mathrm{cm}}\right)$ is the relative three-momentum of the incoming (outgoing) particles in the $\mathrm{cm}$ frame. Here, $s, t$, and $u$ are the usual Mandelstam variables. For the different $\gamma^{*} N \rightarrow \pi N$ channels, we obtain the following amplitudes $\mathcal{A}_{i}$, where we introduce $q_{A}$ as the four-momentum of the incoming photon and $q_{B}$ as the four-momentum of the outgoing pion:

(i) $\gamma^{*} p \rightarrow \pi^{0} p$

$$
\begin{aligned}
& \mathcal{A}_{1}=\frac{e g_{A} M_{N}}{f_{\pi}}\left[\frac{2 P_{A} \cdot \epsilon+q_{A} \cdot \epsilon}{s-M_{N}^{2}}+\frac{2 P_{B} \cdot \epsilon-q_{A} \cdot \epsilon}{u-M_{N}^{2}}\right], \\
& \mathcal{A}_{2}=\frac{e g_{A} M_{N}}{f_{\pi}}\left[\frac{1}{s-M_{N}^{2}}+\frac{1}{u-M_{N}^{2}}\right] ;
\end{aligned}
$$

(ii) $\gamma^{*} p \rightarrow \pi^{+} n$

$$
\begin{aligned}
\mathcal{A}_{1}= & \frac{\sqrt{2} e g_{A} M_{N}}{f_{\pi}}\left[\frac{2 P_{A} \cdot \epsilon+q_{A} \cdot \epsilon}{s-M_{N}^{2}}\right. \\
& \left.+\frac{2\left(P_{A}-P_{B}\right) \cdot \epsilon+q_{A} \cdot \epsilon}{t-m_{\pi}^{2}}\right], \\
\mathcal{A}_{2}= & \frac{\sqrt{2} e g_{A} M_{N}}{f_{\pi}\left(s-M_{N}^{2}\right)}
\end{aligned}
$$

(iii) $\gamma^{*} n \rightarrow \pi^{0} n$

$$
\begin{aligned}
& \mathcal{A}_{1}=0, \\
& \mathcal{A}_{2}=0 ;
\end{aligned}
$$

(iv) $\gamma^{*} n \rightarrow \pi^{-} p$

$$
\begin{aligned}
\mathcal{A}_{1}= & \frac{\sqrt{2} e g_{A} M_{N}}{f_{\pi}}\left[\frac{2 P_{B} \cdot \epsilon-q_{A} \cdot \epsilon}{u-M_{N}^{2}}\right. \\
& \left.-\frac{2\left(P_{A}-P_{B}\right) \cdot \epsilon+q_{A} \cdot \epsilon}{t-m_{\pi}^{2}}\right], \\
\mathcal{A}_{2}= & \frac{\sqrt{2} e g_{A} M_{N}}{f_{\pi}\left(u-M_{N}^{2}\right)} .
\end{aligned}
$$

The analytical expressions shown above were checked with the amplitudes given in Ref. [88]. Analytical expressions for the tree-level $\gamma^{*} N \rightarrow \pi N$ channel of the $\sigma_{L T}\left(\nu, Q^{2}\right)$ and $\sigma_{T T}\left(\nu, Q^{2}\right)$ cross sections are given below (proton channels: $\pi^{+} n$ and $\pi^{0} p$; neutron channel: $\pi^{-} p$ ). We checked that they reproduce the known results in the real-photon limit $[45,87]$. To shorten the final expressions for the cross sections, which are considerably longer for finite $Q^{2}$ than in the real-photon limit, we define the following dimensionless kinematic variables:

$$
\begin{array}{r}
\alpha_{\gamma}=\left(E_{i}^{N}\right)_{\mathrm{cm}} / \sqrt{s}=\frac{s+M_{N}^{2}+Q^{2}}{2 s}, \\
\alpha_{\pi}=\left(E_{f}^{N}\right)_{\mathrm{cm}} / \sqrt{s}=\frac{s+M_{N}^{2}-m_{\pi}^{2}}{2 s}, \\
\beta_{\gamma}=E_{\mathrm{cm}}^{\gamma} / \sqrt{s}=\frac{s-M_{N}^{2}-Q^{2}}{2 s}, \\
\beta_{\pi}=E_{\mathrm{cm}}^{\pi} / \sqrt{s}=\frac{s-M_{N}^{2}+m_{\pi}^{2}}{2 s}, \\
\lambda_{\gamma}=\left|\vec{q}_{i}\right|_{\mathrm{cm}} / \sqrt{s}=\frac{\sqrt{\left(s-M_{N}^{2}-Q^{2}\right)^{2}+4 s Q^{2}}}{2 s}, \\
\lambda_{\pi}=\left|\vec{q}_{f}\right|_{\mathrm{cm}} / \sqrt{s}=\frac{\sqrt{\left(s-M_{N}^{2}+m_{\pi}^{2}\right)^{2}-4 s m_{\pi}^{2}}}{2 s} .
\end{array}
$$


Here, $\left(E_{i}^{N}\right)_{\mathrm{cm}}$ and $\left(E_{f}^{N}\right)_{\mathrm{cm}}$ are the energies of the incoming and outgoing nucleon, $E_{\mathrm{cm}}^{\gamma}$ is the energy of the incoming photon, and $E_{\mathrm{cm}}^{\pi}$ is the energy of the outgoing pion, all in the $\mathrm{cm}$ frame.

$$
\begin{aligned}
\sigma_{T T}^{\left(\pi^{+} n\right)}= & -\frac{e^{2} g_{A}^{2} M_{N}^{2}}{64 \pi f_{\pi}^{2} s^{3}\left(s-M_{N}^{2}\right)^{2} \lambda_{\gamma}^{4}}\left\{4 s \lambda _ { \pi } \lambda _ { \gamma } \left[\left(M_{N}^{2}-s\right)\left(M_{N}^{2}-Q^{2}-s\right)\left(Q^{2}+2 s \beta_{\gamma} \beta_{\pi}\right)+2 s\left(m_{\pi}^{2} Q^{2}-\left(M_{N}^{2}-s\right)\right.\right.\right. \\
& \left.\left.\times\left(M_{N}^{2}+s\left(-1+2 \beta_{\gamma} \beta_{\pi}\right)\right)\right) \lambda_{\gamma}^{2}\right]-2\left(M_{N}^{2}-s\right)\left(M_{N}^{2}-Q^{2}-s\right) \\
& \left.\times\left(Q^{2}+2 s \beta_{\gamma} \beta_{\pi}-2 s \lambda_{\pi} \lambda_{\gamma}\right)\left(Q^{2}+2 s\left(\beta_{\gamma} \beta_{\pi}+\lambda_{\pi} \lambda_{\gamma}\right)\right) \operatorname{arctanh}\left(\frac{2 s \lambda_{\gamma} \lambda_{\pi}}{Q^{2}+2 s \beta_{\gamma} \beta_{\pi}}\right)\right\}
\end{aligned}
$$

$$
\begin{aligned}
\sigma_{T T}^{\left(\pi^{0} p\right)}= & \frac{e^{2} g_{A}^{2} M_{N}^{2} \lambda_{\pi}}{64 \pi f_{\pi}^{2} s\left(s-M_{N}^{2}\right)^{2} \lambda_{\gamma}}\left\{-4 m_{\pi}^{2} Q^{2}+2\left(M_{N}^{2}-s\right)\left(2\left(s-M_{N}^{2}\right)+4 s \beta_{\gamma} \beta_{\pi}+\frac{\left(s-M_{N}^{2}+Q^{2}\right)\left(3\left(M_{N}^{2}-s\right)+2 s \beta_{\gamma} \beta_{\pi}\right)}{s \lambda_{\gamma}^{2}}\right.\right. \\
& \left.+\frac{2 m_{\pi}^{2} Q^{2}\left(s-M_{N}^{2}\right)}{\left(M_{N}^{2}-s\left(1-2 \beta_{\gamma} \beta_{\pi}+2 \lambda_{\pi} \lambda_{\gamma}\right)\right)\left(M_{N}^{2}-s\left(1-2 \beta_{\gamma} \beta_{\pi}-2 \lambda_{\pi} \lambda_{\gamma}\right)\right)}\right)+\frac{M_{N}^{2}-s}{s^{2} \lambda_{\pi} \lambda_{\gamma}^{3}} \\
& \times\left(Q^{2} s\left(4 \lambda_{\gamma}^{2} m_{\pi}^{2}+s\left(-4 \beta_{\gamma}^{2} \beta_{\pi}^{2}+8 \beta_{\gamma} \beta_{\pi}+4 \lambda_{\pi}^{2} \lambda_{\gamma}^{2}-3\right)\right)+3 M_{N}^{6}\right. \\
& +M_{N}^{4}\left(s\left(8 \beta_{\gamma} \beta_{\pi}+2 \lambda_{\gamma}^{2}-9\right)-3 Q^{2}\right)+s^{3}\left(-4 \beta_{\gamma}^{2} \beta_{\pi}^{2}+8 \beta_{\gamma} \beta_{\pi}+2\left(1+2 \lambda_{\pi}^{2}\right) \lambda_{\gamma}^{2}-3\right) \\
& \left.\left.+M_{N}^{2} s\left(Q^{2}\left(6-8 \beta_{\gamma} \beta_{\pi}\right)+s\left(4 \beta_{\gamma}^{2} \beta_{\pi}^{2}-16 \beta_{\gamma} \beta_{\pi}-4\left(1+\lambda_{\pi}^{2}\right) \lambda_{\gamma}^{2}+9\right)\right)\right) \operatorname{arctanh}\left(\frac{2 s \lambda_{\pi} \lambda_{\gamma}}{M_{N}^{2}+s\left(2 \beta_{\gamma} \beta_{\pi}-1\right)}\right)\right\}
\end{aligned}
$$

$$
\begin{aligned}
\sigma_{T T}^{\left(\pi^{-} p\right)}= & \frac{e^{2} g_{A}^{2} M_{N}^{2}}{16 \pi f_{\pi}^{2} s^{2} \lambda_{\gamma}^{3}}\left\{\frac{\lambda_{\pi}}{M_{N}^{4}-2 M_{N}^{2} s\left(1-2 \beta_{\gamma} \beta_{\pi}\right)-s^{2}\left(-4 \beta_{\gamma}^{2} \beta_{\pi}^{2}+4 \beta_{\gamma} \beta_{\pi}+4 \lambda_{\pi}^{2} \lambda_{\gamma}^{2}-1\right)}\right. \\
& \times\left[Q^{2} s\left(-2 \lambda_{\gamma}^{2} m_{\pi}^{2}+4 \beta_{\gamma}^{2} \beta_{\pi}^{2} s-4 \beta_{\gamma} \beta_{\pi} s-4 \lambda_{\pi}^{2} \lambda_{\gamma}^{2} s+s\right)-M_{N}^{6}\right. \\
& +M_{N}^{4}\left(Q^{2}+s\left(3-4 \beta_{\gamma} \beta_{\pi}\right)\right)+M_{N}^{2} s\left(2 Q^{2}\left(2 \beta_{\gamma} \beta_{\pi}-1\right)\right. \\
& \left.\left.+s\left(-4 \beta_{\gamma}^{2} \beta_{\pi}^{2}+8 \beta_{\gamma} \beta_{\pi}+4 \lambda_{\pi}^{2} \lambda_{\gamma}^{2}-3\right)\right)+s^{3}\left(4 \beta_{\gamma}^{2} \beta_{\pi}^{2}-4 \beta_{\gamma} \beta_{\pi}-4 \lambda_{\pi}^{2} \lambda_{\gamma}^{2}+1\right)\right] \\
& +\frac{1}{2 s \lambda_{\gamma}}\left[\left(Q^{4}+4 \beta_{\gamma} \beta_{\pi} Q^{2} s+4 s^{2}\left(\beta_{\gamma}^{2} \beta_{\pi}^{2}-\lambda_{\pi}^{2} \lambda_{\gamma}^{2}\right)\right) \operatorname{arctanh}\left(\frac{2 s \lambda_{\pi} \lambda_{\gamma}}{Q^{2}+2 s \beta_{\gamma} \beta_{\pi}}\right)\right. \\
& +\left(M_{N}^{4}-2 M_{N}^{2}\left(Q^{2}-\lambda_{\gamma}^{2} s+s\right)+s\left(2 Q^{2}\left(1-2 \beta_{\gamma} \beta_{\pi}\right)-4 \beta_{\gamma}^{2} \beta_{\pi}^{2} s+2\left(2 \lambda_{\pi}^{2}-1\right) \lambda_{\gamma}^{2} s+s\right)\right) \\
& \left.\left.\times \operatorname{arctanh}\left(\frac{2 s \lambda_{\pi} \lambda_{\gamma}}{M_{N}^{2}+s\left(2 \beta_{\gamma} \beta_{\pi}-1\right)}\right)\right]\right\}
\end{aligned}
$$

$$
\begin{aligned}
\sigma_{L T}^{\left(\pi^{+} n\right)}= & \frac{e^{2} g_{A}^{2} M_{N}^{3} \lambda_{\pi}}{32 \pi f_{\pi}^{2} Q s^{3}\left(s-M_{N}^{2}\right)^{2} \lambda_{\gamma}^{4}}\left\{2 s \lambda _ { \gamma } \left[\left(M_{N}^{2}-s\right)\left(Q^{2}+2 s \beta_{\gamma}^{2}\right)\left(Q^{2}+2 s \beta_{\gamma} \beta_{\pi}\right)\right.\right. \\
& \left.-4 s\left(\left(M_{N}^{2}-s\right)\left(Q^{2}-2 s\left(\alpha_{\pi}-1\right) \beta_{\gamma}\right)+Q^{2} s \beta_{\gamma} \beta_{\pi}\right) \lambda_{\gamma}^{2}+8 s^{3}\left(\alpha_{\pi}-1\right) \lambda_{\gamma}^{4}\right] \\
& +\frac{s-M_{N}^{2}}{\lambda_{\pi}}\left[\left(Q^{2}+2 s \beta_{\gamma}^{2}\right)\left(Q^{2}+2 s \beta_{\gamma} \beta_{\pi}\right)^{2}+4 s^{2}\left(2\left(\alpha_{\pi}-1\right) \beta_{\gamma}\left(Q^{2}+2 s \beta_{\gamma} \beta_{\pi}\right)\right.\right. \\
& \left.\left.\left.-Q^{2} \lambda_{\pi}^{2}\right) \lambda_{\gamma}^{2}+8 s^{3}\left(\alpha_{\pi}-1\right)^{2} \lambda_{\gamma}^{4}\right] \operatorname{arctanh}\left(\frac{2 s \lambda_{\pi} \lambda_{\gamma}}{Q^{2}+2 s \beta_{\gamma} \beta_{\pi}}\right)\right\}
\end{aligned}
$$




$$
\begin{aligned}
& \sigma_{L T}^{\left(\pi^{0} p\right)}=\frac{e^{2} g_{A}^{2} M_{N}^{3} \lambda_{\pi}}{16 \pi f_{\pi}^{2} Q s\left(s-M_{N}^{2}\right)^{2} \lambda_{\gamma}}\left\{\frac{1}{-2 s\left(M_{N}^{2}+s\left(-1+2 \beta_{\gamma} \beta_{\pi}\right)\right)^{2} \lambda_{\gamma}^{2}+8 s^{3} \lambda_{\pi}^{2} \lambda_{\gamma}^{4}}\right. \\
& \times\left[-3 M_{N}^{8}\left(Q^{2}+2 s \beta_{\gamma}^{2}\right)+2 M_{N}^{4} s^{2}\left(-\left(Q^{2}+2 s \beta_{\gamma}^{2}\right)\left(2 \beta_{\gamma} \beta_{\pi}-3\right)\left(5 \beta_{\gamma} \beta_{\pi}-3\right)+\left(Q^{2}\left(2 \beta_{\gamma} \beta_{\pi}+6 \lambda_{\pi}^{2}-3\right)\right.\right.\right. \\
& \left.\left.+2 s \beta_{\gamma}\left(12 \alpha_{\pi}+2 \beta_{\gamma} \beta_{\pi}-12 \alpha_{\pi} \beta_{\gamma} \beta_{\pi}+4 \beta_{\gamma} \lambda_{\pi}^{2}-3\right)\right) \lambda_{\gamma}^{2}-4 s\left(\alpha_{\pi}^{2}-1\right) \lambda_{\pi}^{4}\right) \\
& +2 M_{N}^{2} s^{3}\left[-\left(Q^{2}+2 s \beta_{\gamma}^{2}\right)\left(2 \beta_{\gamma} \beta_{\pi}-1\right)\left(6+\beta_{\gamma} \beta_{\pi}\left(-9+2 \beta_{\gamma} \beta_{\pi}\right)\right)+\left(Q^{2}\left(3-12 \lambda_{\pi}^{2}+4 \beta_{\gamma} \beta_{\pi}\left(-1+2 \beta_{\gamma} \beta_{\pi}+\lambda_{\pi}^{2}\right)\right)\right.\right. \\
& \left.\left.-2 s \beta_{\gamma}\left(-3+4 \alpha_{\pi}\left(3+2 \beta_{\gamma} \beta_{\pi}\left(\beta_{\gamma} \beta_{\pi}-3\right)\right)+4 \beta_{\gamma}\left(\beta_{\pi}+\left(2-\beta_{\gamma} \beta_{\pi}\right) \lambda_{\pi}^{2}\right)\right)\right) \lambda_{\gamma}^{2}+8 s\left(\left(\alpha_{\pi}-1\right)\left(1+\alpha_{\pi}-2 \beta_{\gamma} \beta_{\pi}\right)+2 \alpha_{\pi} \beta_{\gamma} \lambda_{\pi}^{2}\right) \lambda_{\gamma}^{4}\right] \\
& +s^{4}\left(\left(Q^{2}+2 s \beta_{\gamma}^{2}\right)\left(1-2 \beta_{\gamma} \beta_{\pi}\right)^{2}\left(2 \beta_{\gamma} \beta_{\pi}-3\right)+2\left(Q^{2}\left(-1+6 \lambda_{\pi}^{2}+2 \beta_{\gamma} \beta_{\pi}\left(\left(1-2 \beta_{\gamma} \beta_{\pi}\right)^{2}-2 \lambda_{\pi}^{2}\right)\right)\right.\right. \\
& \left.+2 s \beta_{\gamma}\left(-1+2 \beta_{\gamma} \beta_{\pi}+4\left(-1+\beta_{\gamma} \beta_{\pi}\right)\left(-\alpha_{\pi}+2 \alpha_{\pi} \beta_{\gamma} \beta_{\pi}-\beta_{\gamma} \lambda_{\pi}^{2}\right)\right)\right) \lambda_{\gamma}^{2}-8\left(s\left(\alpha_{\pi}-1\right)\left(\alpha_{\pi}+\left(1-2 \beta_{\gamma} \beta_{\pi}\right)^{2}\right)\right. \\
& \left.\left.\left.+2 \beta_{\gamma}\left(2 s \alpha_{\pi}+Q^{2} \beta_{\pi}\right) \lambda_{\pi}^{2}\right) \lambda_{\gamma}^{4}+32 s\left(\alpha_{\pi}-1\right) \lambda_{\pi}^{2} \lambda_{\gamma}^{6}\right)+2 M_{N}^{6} s\left(Q^{2}\left(6-7 \beta_{\gamma} \beta_{\pi}+\lambda_{\gamma}^{2}\right)+2 s \beta_{\gamma}\left(\beta_{\gamma}\left(6-7 \beta_{\gamma} \beta_{\pi}\right)+\left(1-4 \alpha_{\pi}\right) \lambda_{\gamma}^{2}\right)\right)\right] \\
& +\frac{s-M_{N}^{2}}{4 s^{2} \lambda_{\pi} \lambda_{\gamma}^{3}}\left[\left(Q^{2}+2 s \beta_{\gamma}^{2}\right)\left(3 M_{N}^{2}+s\left(2 \beta_{\gamma} \beta_{\pi}-3\right)\right)\left(M_{N}^{2}+s\left(2 \beta_{\gamma} \beta_{\pi}-1\right)\right)+2 s\left(-M_{N}^{2}\left(Q^{2}+2 s \beta_{\gamma}-8 s \alpha_{\pi} \beta_{\gamma}\right)\right.\right. \\
& \left.\left.\left.+s\left(2 s \beta_{\gamma}\left(1-4 \alpha_{\pi}+4 \alpha_{\pi} \beta_{\gamma} \beta_{\pi}\right)+Q^{2}\left(1-2 \lambda_{\pi}^{2}\right)\right)\right) \lambda_{\gamma}^{2}+8 s^{3}\left(\alpha_{\pi}^{2}-1\right) \lambda_{\gamma}^{4}\right] \operatorname{arctanh}\left(\frac{2 s \lambda_{\pi} \lambda_{\gamma}}{M_{N}^{2}+s\left(2 \beta_{\gamma} \beta_{\pi}-1\right)}\right)\right\}, \\
& \sigma_{L T}^{\left(\pi^{-} p\right)}=\frac{e^{2} g_{A}^{2} M_{N}^{3}}{16 \pi f_{\pi}^{2} Q s^{2} \lambda_{\gamma}^{3}}\left\{\frac{\lambda_{\pi}}{\left(M_{N}^{2}+s\left(2 \beta_{\gamma} \beta_{\pi}-2 \lambda_{\pi} \lambda_{\gamma}-1\right)\right)\left(M_{N}^{2}+s\left(2 \beta_{\gamma} \beta_{\pi}+2 \lambda_{\pi} \lambda_{\gamma}-1\right)\right)}\right. \\
& \times\left[2 \lambda_{\gamma}^{2} s\left(s\left(\left(1-2 \lambda_{\pi}^{2}\right) Q^{2}+2 \beta_{\gamma} s\left(2 \alpha_{\pi}-1\right)\left(2 \beta_{\gamma} \beta_{\pi}-1\right)\right)-M_{N}^{2}\left(Q^{2}+2 \beta_{\gamma} s\left(1-2 \alpha_{\pi}\right)\right)\right)\right. \\
& \left.+\left(Q^{2}+2 \beta_{\gamma}^{2} s\right)\left(M_{N}^{2}+s\left(2 \beta_{\gamma} \beta_{\pi}-1\right)\right)^{2}+8\left(\alpha_{\pi}-1\right) \alpha_{\pi} \lambda_{\gamma}^{4} s^{3}\right]+\frac{1}{2 s \lambda_{\gamma}\left(s-M_{N}^{2}+Q^{2}\right)} \\
& \times\left[\left(4 \lambda_{\gamma}^{2} s^{2}\left(2\left(\alpha_{\pi}-1\right) \beta_{\gamma}\left(Q^{2}+2 \beta_{\gamma} \beta_{\pi} s\right)-\lambda_{\pi}^{2} Q^{2}\right)+\left(Q^{2}+2 \beta_{\gamma}^{2} s\right)\left(Q^{2}+2 \beta_{\gamma} \beta_{\pi} s\right)^{2}\right.\right. \\
& \left.+8\left(\alpha_{\pi}-1\right)^{2} \lambda_{\gamma}^{4} s^{3}\right) \operatorname{arctanh}\left(\frac{2 s \lambda_{\pi} \lambda_{\gamma}}{Q^{2}+2 s \beta_{\gamma} \beta_{\pi}}\right)+\left(\left(Q^{2}+2 \beta_{\gamma}^{2} s\right)\left(M_{N}^{2}+s\left(2 \beta_{\gamma} \beta_{\pi}-1\right)\right)\right. \\
& \times\left(-M_{N}^{2}+2 Q^{2}+s\left(2 \beta_{\gamma} \beta_{\pi}+1\right)\right)+2 \lambda_{\gamma}^{2} s\left(-M_{N}^{2}\left(Q^{2}+2 \beta_{\gamma} s\right)+Q^{4}\right. \\
& \left.\left.+Q^{2} s\left(2\left(2 \alpha_{\pi}-1\right) \beta_{\gamma}-2 \lambda_{\pi}^{2}+1\right)+2 \beta_{\gamma} s^{2}\left(4\left(\alpha_{\pi}-1\right) \beta_{\gamma} \beta_{\pi}+1\right)\right)+8\left(\alpha_{\pi}-1\right)^{2} \lambda_{\gamma}^{4} s^{3}\right) \\
& \left.\left.\times \operatorname{arctanh}\left(\frac{2 s \lambda_{\pi} \lambda_{\gamma}}{M_{N}^{2}+s\left(2 \beta_{\gamma} \beta_{\pi}-1\right)}\right)\right]\right\} \text {, }
\end{aligned}
$$

\section{2. $\Delta$-production channel}

The tree-level $\Delta$-exchange diagram in Fig. 2 of Ref. [30] contributes to the non-Born part of the VVCS amplitudes. The contribution of the $\Delta$ exchange to the VVCS amplitudes can be split into [17]

$$
\begin{gathered}
\bar{S}_{1}^{\Delta \text {-exch. }}\left(\nu, Q^{2}\right)=S_{1}^{\Delta \text {-pole }}\left(\nu, Q^{2}\right)+\tilde{S}_{1}^{\Delta \text {-exch. }}\left(\nu, Q^{2}\right) \\
\nu \bar{S}_{2}^{\Delta \text {-exch. }}\left(\nu, Q^{2}\right)=\nu S_{2}^{\Delta \text {-pole }}\left(\nu, Q^{2}\right)+\widetilde{\nu S_{2}}{ }^{\Delta \text {-exch. }}\left(\nu, Q^{2}\right),
\end{gathered}
$$

and similarly for the unpolarized VVCS amplitudes discussed in Ref. [30]. Here, we introduced the $\Delta$-pole contributions $S_{i}^{\Delta \text {-pole }}$ and the $\Delta$-nonpole contributions $\tilde{S}_{i}^{\Delta \text {-exch. }}$. The former amplitudes feature a pole at the $\Delta(1232)$-production threshold, and thus, are proportional to 


$$
\frac{1}{\left[s-M_{\Delta}^{2}\right]\left[u-M_{\Delta}^{2}\right]}=\frac{1}{4 M_{N}^{2}} \frac{1}{\nu_{\Delta}^{2}-\nu^{2}} .
$$

They can be reconstructed from the dispersion relations in Eq. (5) with the tree-level $\Delta$-production cross sections as input (cf. Fig. 10 in Ref. [30]),

$$
\begin{aligned}
\sigma_{T T}\left(\nu, Q^{2}\right)= & \frac{\pi^{2} \alpha}{M_{N}^{2} M_{+}^{2}|\vec{q}|}\left\{-g_{M}^{2} M_{N}\left(M_{+}+\nu\right)|\vec{q}|^{2}+\frac{g_{E}^{2}(\Delta-\nu)\left(Q^{2}-M_{N} \nu\right)^{2}}{M_{N}}+\frac{g_{C}^{2} Q^{4} s(\Delta-\nu)}{M_{N} M_{\Delta}^{2}}-4 g_{M} g_{E}\left(Q^{2}-M_{N} \nu\right)|\vec{q}|^{2}\right. \\
& \left.-4 g_{M} g_{C} Q^{2}|\vec{q}|^{2}+\frac{2 g_{E} g_{C} Q^{2}\left[-M_{N} M_{\Delta}|\vec{q}|^{2}+s\left(Q^{2}+\Delta \nu\right)\right]}{M_{N} M_{\Delta}}\right\} \delta\left(\nu-\nu_{\Delta}\right) \\
\sigma_{L T}\left(\nu, Q^{2}\right)= & \frac{Q \pi^{2} \alpha}{M_{N}^{2} M_{+}^{2}|\vec{q}|}\left\{\frac{g_{E}^{2}\left(M_{N} \nu-Q^{2}\right)\left[M_{\Delta}\left(M_{N}+\nu\right)-s\right]}{M_{N}}+\frac{g_{C}^{2} Q^{2}\left[M_{N} M_{\Delta}|\vec{q}|^{2}-s\left(Q^{2}+\Delta \nu\right)\right]}{M_{N} M_{\Delta}^{2}}\right. \\
& \left.+g_{M} g_{E} M_{\Delta}|\vec{q}|^{2}-\frac{g_{M} g_{C}\left(Q^{2}-M_{N} \nu\right)|\vec{q}|^{2}}{M_{\Delta}}+\frac{g_{E} g_{C}(\nu-\Delta)\left(M_{N}^{2}|\vec{q}|^{2}-2 Q^{2} s\right)}{M_{N} M_{\Delta}}\right\} \delta\left(\nu-\nu_{\Delta}\right)
\end{aligned}
$$

with $\Delta=M_{\Delta}-M_{N}, M_{+}=M_{\Delta}+M_{N}$, and the Mandelstam variable $s=M_{N}^{2}+2 M_{N} \nu-Q^{2}$. Analytical expressions for the spin structure functions $g_{1}\left(x, Q^{2}\right)$ and $g_{2}\left(x, Q^{2}\right)$ can be constructed from Eq. (3) with the flux factor $K\left(\nu, Q^{2}\right)=|\vec{q}|=\sqrt{\nu^{2}+Q^{2}}$.

In the $\Delta$-nonpole contributions to $S_{1}\left(\nu, Q^{2}\right)$ and $\nu S_{2}\left(\nu, Q^{2}\right)$, the pole in $\nu$ at the $\Delta(1232)$-production threshold has canceled out:

$$
\begin{aligned}
\tilde{S}_{1}^{\Delta-\text { exch. }}\left(\nu, Q^{2}\right)= & \frac{\pi \alpha}{M_{N} M_{+}^{2}}\left[g_{M}^{2} Q_{+}^{2}+g_{E}^{2}\left(\Delta^{2}-3 Q^{2}\right)+\frac{4 g_{C}^{2} Q^{4}}{M_{\Delta}^{2}}-8 g_{M} g_{E} M_{\Delta} \omega_{-}\right. \\
& \left.-\frac{2 g_{M} g_{C} Q^{2}\left(M_{N}-4 M_{\Delta}\right)}{M_{\Delta}}+\frac{2 g_{E} g_{C} Q^{2}\left(3 M_{N}-2 M_{\Delta}\right)}{M_{\Delta}}\right], \\
{\widetilde{\nu S_{2}}}^{\Delta \text {-exch. }}\left(\nu, Q^{2}\right)= & \frac{2 \pi \alpha}{M_{N} M_{+}^{2}}\left[g_{E}^{2} M_{\Delta} \omega_{-}+\frac{g_{M}^{2} M_{N} Q_{+}^{2}}{2}+\frac{g_{C}^{2} Q^{2}\left(Q^{2}-\Delta^{2}\right)}{2 M_{\Delta}}+g_{E} g_{M} M_{\Delta}\left(M_{\Delta} \omega_{+}-4 M_{N} \omega_{-}\right)\right. \\
& \left.-g_{E} g_{C} \Delta\left(2 Q^{2}+M_{N} \omega_{+}\right)+g_{M} g_{C} Q^{2}\left(4 M_{N}-\omega_{+}\right)\right]+\frac{\tilde{S}_{2}^{\Delta-\text { exch. }}\left(\nu, Q^{2}\right)}{\nu}\left[\frac{M_{\Delta}^{2} \omega_{+}^{2}}{M_{N}^{2}}+\nu^{2}\right],
\end{aligned}
$$

with $Q_{+}=\sqrt{\left(M_{\Delta}+M_{N}\right)^{2}+Q^{2}}$ and $\omega_{ \pm}=\left(M_{\Delta}^{2}-M_{N}^{2} \pm\right.$ $\left.Q^{2}\right) / 2 M_{\Delta}$, and the nonpole contribution to $S_{2}\left(\nu, Q^{2}\right)$ :

$$
\tilde{S}_{2}^{\Delta-\text { exch. }}\left(\nu, Q^{2}\right)=-\frac{2 \pi \alpha M_{N} \nu}{M_{\Delta} M_{+}^{2}}\left[g_{M}+g_{E}\right] g_{C} .
$$

These amplitudes, to the contrary, are not described by the tree-level $\Delta$-production cross sections in the standard dispersive approach [17]. This peculiarity has been previously missed, e.g., in the calculation of the $\Delta$-exchange contribution to the hydrogen hyperfine splitting in Ref. [89]. The importance of including the $\Delta$-nonpole contribution is also evident when considering the BC sum rule in Eq. (14). The $\Delta$-pole terms by themselves violate the $\mathrm{BC}$ sum rule, but cancel exactly with the $\Delta$-nonpole terms:

$$
\lim _{\nu \rightarrow 0} \nu S_{2}^{\Delta \text {-pole }}\left(\nu, Q^{2}\right)+\lim _{\nu \rightarrow 0}{\widetilde{\nu S_{2}}}^{\Delta \text {-exch. }}\left(\nu, Q^{2}\right)=0 .
$$

\section{APPENDIX C: POLARIZABILITIES AT $Q^{2}=0$}

In this section, we give analytical expressions for the polarizabilities and their slopes at $Q^{2}=0$. In particular, we give the HB expansion of the $\pi N$-loop contributions and the $\Delta$-exchange contributions. The complete expressions, also for the $\pi \Delta$-loop contributions, can be found in the Supplemental Material [38]. Recall that $I_{A}(0)=I_{1}(0)=$ $\bar{d}_{2}(0)=0$ and $\left.\frac{\mathrm{d} \bar{d}_{2}\left(Q^{2}\right)}{\mathrm{d} Q^{2}}\right|_{Q^{2}=0}=0$.

\section{1. $\pi N$-loop contribution}

Here, we give analytical expressions for the $\pi N$-loop contributions to the proton and neutron spin polarizabilities, expanded in powers of $\mu=m_{\pi} / M_{N}$, viz. the HB expansion. Note that we choose to expand here to a high order in $\mu$; the strict HB expansion would only retain the leading term in an analogous NLO calculation. 
(i) Polarizabilities at $Q^{2}=0$ :

$$
\begin{gathered}
\gamma_{0 p}=\frac{e^{2} g_{A}^{2}}{96 \pi^{3} f_{\pi}^{2} m_{\pi}^{2}}\left\{1-\frac{21 \pi \mu}{8}-\left(\frac{59}{2}+26 \log \mu\right) \mu^{2}+\frac{1875 \pi \mu^{3}}{64}+3\left(\frac{3}{2}+26 \log \mu\right) \mu^{4}+\cdots\right\}, \\
\gamma_{0 n}=\frac{e^{2} g_{A}^{2}}{48 \pi^{3} f_{\pi}^{2} m_{\pi}^{2}}\left\{\frac{1}{2}-\frac{9 \pi \mu}{16}-2 \mu^{2} \log \mu+\frac{75 \pi \mu^{3}}{128}-\frac{3 \mu^{4}}{4}+\cdots\right\}, \\
\delta_{L T p}=\frac{e^{2} g_{A}^{2}}{192 \pi^{3} f_{\pi}^{2} m_{\pi}^{2}}\left\{1-\frac{9 \pi \mu}{8}+\left(\frac{13}{2}-2 \log \mu\right) \mu^{2}-\frac{465 \pi \mu^{3}}{64}-\left(\frac{47}{2}+42 \log \mu\right) \mu^{4}+\cdots\right\}, \\
\delta_{L T n}=\frac{e^{2} g_{A}^{2}}{96 \pi^{3} f_{\pi}^{2} m_{\pi}^{2}}\left\{\frac{1}{2}+\frac{3 \pi \mu}{16}+(1+2 \log \mu) \mu^{2}-\frac{105 \pi \mu^{3}}{128}+\frac{5 \mu^{4}}{4}+\cdots\right\}, \\
\delta_{L T n}=\frac{e^{2} g_{A}^{2}}{96 \pi^{3} f_{\pi}^{2} m_{\pi}^{2}}\left\{\frac{1}{2}+\frac{3 \pi \mu}{16}+(1+2 \log \mu) \mu^{2}-\frac{105 \pi \mu^{3}}{128}+\frac{5 \mu^{4}}{4}+\cdots\right\}, \\
\bar{\gamma}_{0 p}=\frac{e^{2} g_{A}^{2}}{16 \pi^{3} f_{\pi}^{2} m_{\pi}^{4}}\left\{\frac{4}{45}-\frac{3 \pi \mu}{16}+\frac{14 \mu^{2}}{5}-\frac{1813 \pi \mu^{3}}{384}-\frac{192}{5}(1+\log \mu) \mu^{4}+\frac{80703 \pi \mu^{5}}{2048}+\cdots\right\}, \\
\bar{\gamma}_{0 n}=\frac{e^{2} g_{A}^{2}}{16 \pi^{3} f_{\pi}^{2} m_{\pi}^{4}}\left\{\frac{4}{45}-\frac{5 \pi \mu}{48}+\frac{4 \mu^{2}}{5}-\frac{245 \pi \mu^{3}}{384}-\frac{32 \mu^{4} \log \mu}{15}+\frac{1323 \pi \mu^{5}}{2048}+\cdots\right\} .
\end{gathered}
$$

(ii) Slopes of polarizabilities at $Q^{2}=0$ :

$$
\begin{aligned}
& \left.\frac{\mathrm{d} \gamma_{0 p}\left(Q^{2}\right)}{\mathrm{d} Q^{2}}\right|_{Q^{2}=0}=\frac{e^{2} g_{A}^{2}}{1440 \pi^{3} f_{\pi}^{2} m_{\pi}^{4}}\left\{2-\frac{45 \pi \mu}{4}+223 \mu^{2}-\frac{28515 \pi \mu^{3}}{64}-9\left(\frac{1953}{4}+449 \log \mu\right) \mu^{4}+\frac{570255 \pi \mu^{5}}{128}+\cdots\right\}, \\
& \left.\frac{\mathrm{d} \gamma_{0 n}\left(Q^{2}\right)}{\mathrm{d} Q^{2}}\right|_{Q^{2}=0}=\frac{e^{2} g_{A}^{2}}{1440 \pi^{3} f_{\pi}^{2} m_{\pi}^{4}}\left\{2-\frac{81 \pi \mu}{8}+94 \mu^{2}-\frac{2535 \pi \mu^{3}}{32}-3(1+90 \log \mu) \mu^{4}+\frac{84315 \pi \mu^{5}}{1024}+\cdots\right\}, \\
& \left.\frac{\mathrm{d} \delta_{L T p}\left(Q^{2}\right)}{\mathrm{d} Q^{2}}\right|_{Q^{2}=0}=\frac{e^{2} g_{A}^{2}}{2880 \pi^{3} f_{\pi}^{2} m_{\pi}^{4}}\left\{-\frac{5}{2}-\frac{27 \pi \mu}{32}+20 \mu^{2}-\frac{5865 \pi \mu^{3}}{256}+3\left(\frac{617}{4}+36 \log \mu\right) \mu^{4}-\frac{2056845 \pi \mu^{5}}{4096}+\cdots\right\}, \\
& \left.\frac{\mathrm{d} \delta_{L T n}\left(Q^{2}\right)}{\mathrm{d} Q^{2}}\right|_{Q^{2}=0}=\frac{e^{2} g_{A}^{2}}{1440 \pi^{3} f_{\pi}^{2} m_{\pi}^{4}}\left\{-\frac{5}{4}-\frac{81 \pi \mu}{64}-11 \mu^{2}+\frac{10005 \pi \mu^{3}}{512}+\frac{15}{8}(11+48 \log \mu) \mu^{4}-\frac{267015 \pi \mu^{5}}{8192}+\cdots\right\}, \\
& \left.\frac{\mathrm{d} I_{A p}\left(Q^{2}\right)}{\mathrm{d} Q^{2}}\right|_{Q^{2}=0}=\frac{g_{A}^{2}}{96 \pi^{2} f_{\pi}^{2} \mu^{2}}\left\{1-\frac{15 \pi \mu}{4}-\frac{1}{2}(115+88 \log \mu) \mu^{2}+\frac{1839 \pi \mu^{3}}{32}+5(5+34 \log \mu) \mu^{4}+\cdots\right\}, \\
& \left.\frac{\mathrm{d} I_{A n}\left(Q^{2}\right)}{\mathrm{d} Q^{2}}\right|_{Q^{2}=0}=\frac{g_{A}^{2}}{48 \pi^{2} f_{\pi}^{2} \mu^{2}}\left\{\frac{1}{2}-\frac{11 \pi \mu}{8}-\frac{1}{4}(1+20 \log \mu) \mu^{2}+\frac{99 \pi \mu^{3}}{64}-\frac{25 \mu^{4}}{12}+\cdots\right\}, \\
& \left.\frac{\mathrm{d} I_{1 p}\left(Q^{2}\right)}{\mathrm{d} Q^{2}}\right|_{Q^{2}=0}=\frac{g_{A}^{2}}{96 \pi^{2} f_{\pi}^{2} \mu}\left\{\frac{3 \pi}{8}+2(4+3 \log \mu) \mu-\frac{537 \pi \mu^{2}}{64}-\frac{1}{2}(15+56 \log \mu) \mu^{3}+\cdots\right\}, \\
& \left.\frac{\mathrm{d} I_{1 n}\left(Q^{2}\right)}{\mathrm{d} Q^{2}}\right|_{Q^{2}=0}=\frac{g_{A}^{2}}{48 \pi^{2} f_{\pi}^{2} \mu}\left\{-\frac{\pi}{16}+\frac{1}{4}(3+4 \log \mu) \mu-\frac{57 \pi \mu^{2}}{128}+\frac{2 \mu^{3}}{3}+\cdots\right\},
\end{aligned}
$$




$$
\begin{aligned}
& \left.\frac{\mathrm{d} \bar{\gamma}_{0 p}\left(Q^{2}\right)}{\mathrm{d} Q^{2}}\right|_{Q^{2}=0}=\frac{e^{2} g_{A}^{2}}{16 \pi^{3} f_{\pi}^{2} m_{\pi}^{6}}\left\{\frac{1}{105}-\frac{23 \pi \mu}{256}+\frac{377 \mu^{2}}{210}-\frac{15551 \pi \mu^{3}}{6144}+\frac{3371 \mu^{4}}{105}-\frac{1640457 \pi \mu^{5}}{32768}+\ldots\right\}, \\
& \left.\frac{\mathrm{d} \bar{\gamma}_{0 n}\left(Q^{2}\right)}{\mathrm{d} Q^{2}}\right|_{Q^{2}=0}=\frac{e^{2} g_{A}^{2}}{16 \pi^{3} f_{\pi}^{2} m_{\pi}^{6}}\left\{\frac{1}{105}-\frac{153 \pi \mu}{1792}+\frac{69 \mu^{2}}{70}-\frac{4615 \pi \mu^{3}}{6144}+\frac{172 \mu^{4}}{35}-\frac{120897 \pi \mu^{5}}{32768}+\ldots\right\} .
\end{aligned}
$$

\section{2. $\Delta$-exchange contribution}

Here, we give analytical expressions for the tree-level $\Delta$-exchange contributions to the nucleon spin polarizabilities and their slopes at $Q^{2}=0$. Note that the $\Delta$-exchange contributes equally to proton and neutron polarizabilities. Recall that for the magnetic $\gamma^{*} N \Delta$ coupling we introduced a dipole form factor to mimic vector-meson dominance: $g_{M} \rightarrow g_{M} /\left(1+Q^{2} / \Lambda^{2}\right)^{2}$.

(i) Polarizabilities at $Q^{2}=0$ :

$$
\begin{gathered}
\gamma_{0}=-\frac{e^{2}}{4 \pi M_{+}^{2}}\left(\frac{g_{M}^{2}}{\Delta^{2}}+\frac{g_{E}^{2}}{M_{+}^{2}}-\frac{4 g_{M} g_{E}}{M_{+} \Delta}\right), \\
\delta_{L T}=\frac{e^{2} M_{\Delta}}{4 \pi M_{+}^{3}}\left(\frac{g_{E}^{2}}{M_{N} M_{+}}+\frac{g_{M} g_{E}}{\Delta M_{N}}-\frac{g_{E} g_{C}}{M_{\Delta}^{2}}\right), \\
\bar{\gamma}_{0}=\frac{e^{2} M_{N}^{2}}{\pi \Delta^{2} M_{+}^{4}}\left(-\frac{g_{M}^{2}}{\Delta^{2}}+\frac{g_{E}^{2}}{M_{+}^{2}}+\frac{4 g_{M} g_{E}}{\Delta M_{+}}\right) .
\end{gathered}
$$

(ii) Slopes of polarizabilities at $Q^{2}=0$ :

$$
\begin{aligned}
& \left.\frac{\mathrm{d} \gamma_{0}\left(Q^{2}\right)}{\mathrm{d} Q^{2}}\right|_{Q^{2}=0}=-\frac{e^{2}}{\pi M_{+}^{2} \Delta}\left(\frac{g_{M}^{2}}{\Delta}\left[\frac{1}{4 \Delta^{2}}-\frac{1}{\Delta M_{+}}+\frac{1}{2 M_{+}^{2}}\right]-\frac{1}{\Lambda^{2}} \frac{g_{M}^{2}}{\Delta}+\frac{g_{E}^{2}}{2 M_{+}^{2}}\left[\frac{1}{2 \Delta}-\frac{3}{M_{+}}\right]\right. \\
& \left.-\frac{g_{M} g_{E}}{M_{+}}\left[\frac{1}{\Delta^{2}}-\frac{5}{\Delta M_{+}}+\frac{1}{M_{+}^{2}}\right]+\frac{1}{\Lambda^{2}} \frac{2 g_{M} g_{E}}{M_{+}}+\frac{2 g_{M} g_{C}}{\Delta M_{+}^{2}}-\frac{g_{E} g_{C}}{M_{+}^{3}}\right), \\
& \left.\frac{\mathrm{d} \delta_{L T}\left(Q^{2}\right)}{\mathrm{d} Q^{2}}\right|_{Q^{2}=0}=\frac{e^{2} M_{\Delta} \Delta}{4 \pi M_{N} M_{+}^{2}}\left(\frac{g_{E}^{2}}{\Delta^{2} M_{+}^{2}}\left[\frac{1}{\Delta}-\frac{4}{M_{+}}\right]-\frac{g_{C}^{2}}{\Delta M_{\Delta}^{2} M_{+}^{2}}+\frac{g_{M} g_{E}}{\Delta^{2} M_{+}}\left[\frac{1}{\Delta^{2}}-\frac{3}{\Delta M_{+}}+\frac{1}{M_{+}^{2}}\right]\right. \\
& \left.-\frac{2}{\Lambda^{2}} \frac{g_{M} g_{E}}{\Delta^{2} M_{+}}+\frac{g_{M} g_{C}}{\Delta M_{\Delta}^{2}}\left[\frac{1}{2 \Delta^{2}}-\frac{2}{\Delta M_{+}}+\frac{1}{2 M_{+}^{2}}\right]-\frac{g_{E} g_{C}}{2 M_{\Delta}^{2} M_{+}^{2}}\left[\frac{7}{\Delta}+\frac{1}{M_{+}}\right]\right), \\
& \left.\frac{\mathrm{d} I_{A}\left(Q^{2}\right)}{\mathrm{d} Q^{2}}\right|_{Q^{2}=0}=-\frac{M_{N}^{2}}{M_{+}^{2}}\left(\frac{g_{M}^{2}}{2 \Delta^{2}}+\frac{g_{E}^{2}}{M_{N} M_{+}}-\frac{2 g_{M} g_{E}}{\Delta M_{+}}-\frac{g_{E} g_{C}}{M_{\Delta} M_{+}}\right), \\
& \left.\frac{\mathrm{d} I_{1}\left(Q^{2}\right)}{\mathrm{d} Q^{2}}\right|_{Q^{2}=0}=-\frac{M_{\Delta} M_{N}^{2}}{2 M_{+}^{3}}\left(\frac{g_{E}^{2}}{M_{N} M_{\Delta}}-\frac{g_{M} g_{E}}{\Delta M_{N}}-\frac{g_{E} g_{C}}{M_{\Delta}^{2}}\right), \\
& \left.\frac{\mathrm{d} \bar{\gamma}_{0}\left(Q^{2}\right)}{\mathrm{d} Q^{2}}\right|_{Q^{2}=0}=\frac{e^{2} M_{N}^{2}}{\pi \Delta^{3} M_{+}^{6}}\left(\frac{g_{M}^{2}}{\Delta}\left[\frac{3 M_{\Delta}^{2}+2 M_{\Delta} M_{N}-9 M_{N}^{2}}{\Delta^{2}}+\frac{4 M_{+}^{2}}{\Lambda^{2}}\right]-4 g_{M} g_{E}\left[\frac{5 M_{\Delta}^{2}-9 M_{N}^{2}}{M_{+} \Delta^{2}}+\frac{2 M_{+}}{\Lambda^{2}}\right]\right. \\
& \left.+\frac{g_{E}^{2}}{\Delta M_{+}}\left[7 M_{\Delta}-9 M_{N}\right]-\frac{8 g_{M} g_{C}}{\Delta}+\frac{4 g_{E} g_{C}}{M_{+}}\right) .
\end{aligned}
$$


[1] D. Drechsel, B. Pasquini, and M. Vanderhaeghen, Dispersion relations in real and virtual Compton scattering, Phys. Rep. 378, 99 (2003).

[2] S. E. Kuhn, J.-P. Chen, and E. Leader, Spin structure of the nucleon-Status and recent results, Prog. Part. Nucl. Phys. 63, 1 (2009).

[3] F. Hagelstein, R. Miskimen, and V. Pascalutsa, Nucleon polarizabilities: From Compton scattering to hydrogen atom, Prog. Part. Nucl. Phys. 88, 29 (2016).

[4] B. Pasquini and M. Vanderhaeghen, Dispersion theory in electromagnetic interactions, Annu. Rev. Nucl. Part. Sci. 68, 75 (2018).

[5] S. Gerasimov, A sum rule for magnetic moments and the damping of the nucleon magnetic moment in nuclei, Sov. J. Nucl. Phys. 2, 430 (1966).

[6] S. Drell and A. C. Hearn, Exact Sum Rule for Nucleon Magnetic Moments, Phys. Rev. Lett. 16, 908 (1966).

[7] J. S. Schwinger, Source theory viewpoints in deep inelastic scattering, Proc. Natl. Acad. Sci. U.S.A. 72, 1 (1975).

[8] J. S. Schwinger, Source theory viewpoints in deep inelastic scattering, Acta Phys. Austriaca Suppl. 14, 471 (1975).

[9] J. Schwinger, Source theory discussion of deep inelastic scattering with polarized particles, Proc. Natl. Acad. Sci. U.S.A. 72, 1559 (1975).

[10] A. M. Baldin, Polarizability of nucleons, Nucl. Phys. 18, 310 (1960).

[11] M. Gell-Mann, M. L. Goldberger, and W. E. Thirring, Use of causality conditions in quantum theory, Phys. Rev. 95, 1612 (1954).

[12] A. Pineda, Leading chiral logarithms to the hyperfine splitting of the hydrogen and muonic hydrogen, Phys. Rev. C 67, 025201 (2003).

[13] C. Peset and A. Pineda, The two-photon exchange contribution to muonic hydrogen from chiral perturbation theory, Nucl. Phys. B887, 69 (2014).

[14] C. Peset and A. Pineda, Model-independent determination of the two-photon exchange contribution to hyperfine splitting in muonic hydrogen, J. High Energy Phys. 04 (2017) 060.

[15] F. Hagelstein and V. Pascalutsa, Proton structure in the hyperfine splitting of muonic hydrogen, Proc. Sci., CD15 (2016) 077 [arXiv:1511.04301].

[16] F. Hagelstein, Exciting nucleons in Compton scattering and hydrogen-like atoms, Ph.D. thesis, Mainz University, Inst. Kernphys., 2017.

[17] F. Hagelstein, $\Delta(1232)$-Resonance in the hydrogen spectrum, Few Body Syst. 59, 93 (2018).

[18] Y. Prok et al. (CLAS Collaboration), Moments of the spin structure functions $g_{1}^{p}$ and $g_{1}^{d}$ for $0.05<Q^{2}<3.0 \mathrm{GeV}^{2}$, Phys. Lett. B 672, 12 (2009).

[19] H. Dutz et al. (GDH Collaboration), First Measurement of the Gerasimov-Drell-Hearn Sum Rule for ${ }^{1} \mathrm{H}$ from $0.7 \mathrm{GeV}$ to $1.8 \mathrm{GeV}$ at ELSA, Phys. Rev. Lett. 91, 192001 (2003).

[20] M. Amarian et al. (Jefferson Lab E94010 Collaboration), Measurement of the Generalized Forward Spin Polarizabilities of the Neutron, Phys. Rev. Lett. 93, 152301 (2004).

[21] M. Amarian et al., The $Q^{2}$ Evolution of the Generalized Gerasimov-Drell-Hearn Integral for the Neutron Using a ${ }^{3}$ He Target, Phys. Rev. Lett. 89, 242301 (2002).
[22] M. Amarian et al. (Jefferson Lab E94-010 Collaboration), $Q^{2}$ Evolution of the Neutron Spin Structure Moments Using a ${ }^{3} \mathrm{He}$ Target, Phys. Rev. Lett. 92, 022301 (2004).

[23] A. Deur et al., Experimental Determination of the Evolution of the Bjorken Integral at Low $Q^{2}$, Phys. Rev. Lett. 93, 212001 (2004).

[24] K. Slifer, Low $Q^{2}$ measurement of $g_{2}^{p}$ and the $\delta_{L T}$ spin polarizability, AIP Conf. Proc. 1155, 125 (2009).

[25] P. Solvignon et al. (E01-012 Collaboration), Moments of the neutron $g_{2}$ structure function at intermediate $Q^{2}$, Phys. Rev. C 92, 015208 (2015).

[26] A. Deur, Experimental studies at low $Q^{2}$ of the spin structure of the nucleon at Jefferson Lab, Proc. Sci., CD2018 (2019) 005 [arXiv:1903.05661].

[27] V. Sulkosky et al. (Jefferson Lab E97-110 Collaboration), Measurement of the ${ }^{3} \mathrm{He}$ spin-structure functions and of neutron $\left({ }^{3} \mathrm{He}\right)$ spin-dependent sum rules at $0.035 \leq Q^{2} \leq$ $0.24 \mathrm{GeV}^{2}$, Phys. Lett. B 805, 135428 (2020).

[28] K. Adhikari et al. (CLAS Collaboration), Measurement of the $Q^{2}$ Dependence of the Deuteron Spin Structure Function $g_{1}$ and its Moments at Low $Q^{2}$ with CLAS, Phys. Rev. Lett. 120, 062501 (2018).

[29] V. Lensky, F. Hagelstein, A. Hiller Blin, and V. Pascalutsa, Comment on "Measurement of the $Q^{2}$ dependence of the deuteron spin structure function $g_{1}$ and its moments at low $Q^{2}$ with CLAS", arXiv:1806.03219.

[30] J. M. Alarcón, F. Hagelstein, V. Lensky, and V. Pascalutsa, Forward doubly-virtual Compton scattering off the nucleon in chiral perturbation theory: the subtraction function and moments of unpolarized structure functions, Phys. Rev. D 102, 014006 (2020).

[31] V. Bernard, E. Epelbaum, H. Krebs, and U.-G. Meißner, New insights into the spin structure of the nucleon, Phys. Rev. D 87, 054032 (2013).

[32] V. Lensky, J. M. Alarcón, and V. Pascalutsa, Moments of nucleon structure functions at next-to-leading order in baryon chiral perturbation theory, Phys. Rev. C 90, 055202 (2014).

[33] V. Pascalutsa and M. Vanderhaeghen, Chiral effective-field theory in the $\Delta(1232)$ region. I: Pion electroproduction on the nucleon, Phys. Rev. D 73, 034003 (2006).

[34] V. Pascalutsa and M. Vanderhaeghen, Electromagnetic Nucleon-to-Delta Transition in Chiral Effective Field Theory, Phys. Rev. Lett. 95, 232001 (2005).

[35] T. R. Hemmert, B. R. Holstein, and J. Kambor, Systematic $1 / \mathrm{M}$ expansion for spin $3 / 2$ particles in baryon chiral perturbation theory, Phys. Lett. B 395, 89 (1997).

[36] V. Pascalutsa and D. R. Phillips, Effective theory of the $\Delta(1232)$ in Compton scattering off the nucleon, Phys. Rev. C 67, 055202 (2003).

[37] V. Pascalutsa, M. Vanderhaeghen, and S. N. Yang, Electromagnetic excitation of the $\Delta(1232)$-resonance, Phys. Rep. 437, 125 (2007).

[38] See Supplemental Material at http://link.aps.org/ supplemental/10.1103/PhysRevD.102.114026 for the analytic expressions of the considered contributions to the polarizabilities and their slopes at the real-photon point, as well as their HB expansion, provided as a Mathematica 12 notebook.

[39] F. E. Low, Scattering of light of very low frequency by systems of spin 1/2, Phys. Rev. 96, 1428 (1954). 
[40] M. Gell-Mann and M. L. Goldberger, Scattering of lowenergy photons by particles of spin 1/2, Phys. Rev. 96, 1433 (1954).

[41] A. M. Harun ar-Rashid, A simple derivation of Schwinger's sum rule for spin dependent structure functions, Nuovo Cimento A 33, 447 (1976).

[42] F. Hagelstein and V. Pascalutsa, Dissecting the Hadronic Contributions to $(g-2)_{\mu}$ by Schwinger's Sum Rule, Phys. Rev. Lett. 120, 072002 (2018).

[43] V. Lensky, V. Pascalutsa, M. Vanderhaeghen, and C. Kao, Spin-dependent sum rules connecting real and virtual Compton scattering verified, Phys. Rev. D 95, 074001 (2017).

[44] H. Burkhardt and W. N. Cottingham, Sum rules for forward virtual Compton scattering, Ann. Phys. (N.Y.) 56, 453 (1970).

[45] V. Lensky and V. Pascalutsa, Predictive powers of chiral perturbation theory in Compton scattering off protons, Eur. Phys. J. C 65, 195 (2010).

[46] K. A. Olive et al. (Particle Data Group), Review of particle physics, Chin. Phys. C 38, 090001 (2014).

[47] H. Grießhammer, J. McGovern, D. Phillips, and G. Feldman, Using effective field theory to analyse low-energy Compton scattering data from protons and light nuclei, Prog. Part. Nucl. Phys. 67, 841 (2012).

[48] H. W. Grießhammer, J. A. McGovern, and D. R. Phillips, Nucleon polarisabilities at and beyond physical pion masses, Eur. Phys. J. A 52, 139 (2016).

[49] E. Epelbaum, H. Krebs, and U.-G. Meißner, Improved chiral nucleon-nucleon potential up to next-to-next-to-next-toleading order, Eur. Phys. J. A 51, 53 (2015).

[50] E. Epelbaum, H. Krebs, and U.-G. Meißner, Precision Nucleon-Nucleon Potential at Fifth Order in the Chiral Expansion, Phys. Rev. Lett. 115, 122301 (2015).

[51] C. W. Kao, T. Spitzenberg, and M. Vanderhaeghen, Burkhardt-Cottingham sum rule and forward spin polarizabilities in heavy baryon chiral perturbation theory, Phys. Rev. D 67, 016001 (2003).

[52] C.-W. Kao, D. Drechsel, S. Kamalov, and M. Vanderhaeghen, Higher moments of nucleon spin structure functions in heavy baryon chiral perturbation theory and in a resonance model, Phys. Rev. D 69, 056004 (2004).

[53] T. Becher and H. Leutwyler, Baryon chiral perturbation theory in manifestly Lorentz invariant form, Eur. Phys. J. C 9, 643 (1999).

[54] J. Gasser, M. E. Sainio, and A. Švarc, Nucleons with chiral loops, Nucl. Phys. B307, 779 (1988).

[55] L. S. Geng, J. Martin Camalich, L. Alvarez-Ruso, and M. J. Vicente Vacas, Leading SU(3)-Breaking Corrections to the Baryon Magnetic Moments in Chiral Perturbation Theory, Phys. Rev. Lett. 101, 222002 (2008).

[56] C. E. Carlson, V. Nazaryan, and K. Griffioen, Proton structure corrections to electronic and muonic hydrogen hyperfine splitting, Phys. Rev. A 78, 022517 (2008).

[57] R. Zielinski, The g2p experiment: A measurement of the proton's spin structure functions, $\mathrm{Ph}$. D. thesis, New Hampshire University, 2010.

[58] N. Guler et al. (CLAS Collaboration), Precise determination of the deuteron spin structure at low to moderate $Q^{2}$ with
CLAS and extraction of the neutron contribution, Phys. Rev. C 92, 055201 (2015).

[59] V. Bernard, N. Kaiser, and U.-G. Meißner, Chiral dynamics in nucleons and nuclei, Int. J. Mod. Phys. E 04, 193 (1995).

[60] V. Bernard, T. R. Hemmert, and U.-G. Meißner, Spin structure of the nucleon at low energies, Phys. Rev. D 67, 076008 (2003).

[61] D. Drechsel, S. S. Kamalov, and L. Tiator, The GDH sum rule and related integrals, Phys. Rev. D 63, 114010 (2001).

[62] D. Drechsel, O. Hanstein, S. S. Kamalov, and L. Tiator, A unitary isobar model for pion photo- and electroproduction on the proton up to $1 \mathrm{GeV}$, Nucl. Phys. A645, 145 (1999).

[63] L. Tiator (private communication) (2020).

[64] O. Gryniuk, F. Hagelstein, and V. Pascalutsa, Evaluation of the forward Compton scattering off protons: II. Spindependent amplitude and observables, Phys. Rev. D 94, 034043 (2016).

[65] T. R. Hemmert, B. R. Holstein, and J. Kambor, $\Delta$ (1232) and the polarizabilities of the nucleon, Phys. Rev. D 55, 5598 (1997).

[66] V. Pascalutsa and R. Timmermans, Field theory of nucleon to higher-spin baryon transitions, Phys. Rev. C 60, 042201 (1999).

[67] V. Pascalutsa, Quantization of an interacting spin-3/2 field and the Delta isobar, Phys. Rev. D 58, 096002 (1998).

[68] H. Krebs, Double virtual Compton scattering and spinstructure of the nucleon, Proc. Sci. CD2018 (2019) 031.

[69] D. Drechsel, S. Kamalov, and L. Tiator, Unitary isobar model-MAID2007, Eur. Phys. J. A 34, 69 (2007).

[70] P. J. Mohr, B. N. Taylor, and D. B. Newell, CODATA recommended values of the fundamental physical constants: 2010, Rev. Mod. Phys. 84, 1527 (2012).

[71] N. Kochelev and Y. Oh, Axial anomaly and the $\delta_{L T}$ puzzle, Phys. Rev. D 85, 016012 (2012).

[72] J. Ahrens et al. (GDH and A2 Collaboration), First Measurement of the Gerasimov-Drell-Hearn Integral for ${ }^{1} \mathrm{H}$ from 200 to $800 \mathrm{MeV}$, Phys. Rev. Lett. 87, 022003 (2001).

[73] K. Helbing (GDH Collaboration), Experimental verification of the GDH sum rule at ELSA and MAMI, Nucl. Phys. B, Proc. Suppl. 105, 113 (2002).

[74] R. Bradford, A. Bodek, H. S. Budd, and J. Arrington, A new parameterization of the nucleon elastic form-factors, Nucl. Phys. B, Proc. Suppl. 159, 127 (2006).

[75] J.D. Bjorken, Applications of the chiral $\mathrm{U}(6) \times \mathrm{U}(6)$ algebra of current densities, Phys. Rev. 148, 1467 (1966).

[76] J. D. Bjorken, Inelastic scattering of polarized leptons from polarized nucleons, Phys. Rev. D 1, 1376 (1970).

[77] V. Bernard, T. R. Hemmert, and U.-G. Meißner, Novel analysis of chiral loop effects in the generalized GerasimovDrell-Hearn sum rule, Phys. Lett. B 545, 105 (2002).

[78] A. Deur et al., Experimental study of isovector spin sum rules, Phys. Rev. D 78, 032001 (2008).

[79] R. Jaffe, $g_{2}$-The nucleon's other spin-dependent structure function, Comments Nucl. Part. Phys. 19, 239 (1990).

[80] E. V. Shuryak and A. Vainshtein, Theory of power corrections to deep inelastic scattering in quantum chromodynamics: (II). $Q^{-4}$ effects; Polarized target, Nucl. Phys. B201, 141 (1982). 
[81] S. Wandzura and F. Wilczek, Sum rules for spin dependent electroproduction: Test of relativistic constituent quarks, Phys. Lett. B 72, 195 (1977).

[82] B. W. Filippone and X.-D. Ji, The spin structure of the nucleon, Adv. Nucl. Phys. 26, 1 (2001).

[83] M. Burkardt, The $g_{2}$ structure function, AIP Conf. Proc. 1155, 26 (2009).

[84] A. Deur, S. J. Brodsky, and G. F. de Téramond, The spin structure of the nucleon, Rep. Prog. Phys. 82, 076201 (2019).

[85] B. Pasquini, P. Pedroni, and D. Drechsel, Higher order forward spin polarizability, Phys. Lett. B 687, 160 (2010).
[86] B. R. Holstein, D. Drechsel, B. Pasquini, and M. Vanderhaeghen, Higher order polarizabilities of the proton, Phys. Rev. C 61, 034316 (2000).

[87] B. R. Holstein, V. Pascalutsa, and M. Vanderhaeghen, Sum rules for magnetic moments and polarizabilities in QED and chiral effective-field theory, Phys. Rev. D 72, 094014 (2005).

[88] B. Pasquini, D. Drechsel, and L. Tiator, Invariant amplitudes for pion electroproduction, Eur. Phys. J. A 34, 387 (2007).

[89] A. J. Buchmann, Non-spherical proton shape and hydrogen hyperfine splitting, Can. J. Phys. 87, 773 (2009). 